\title{
Near-IR photometry of disk galaxies: Search for nuclear isophotal twist and double bars
}

\author{
B. Jungwiert ${ }^{1,2}$, F. Combes ${ }^{1}$, and D.J. Axon ${ }^{3}$ \\ 1 DEMIRM, Observatoire de Paris, 61 Avenue de l'Observatoire, 75014 Paris, France \\ 2 Astronomical Institute, Academy of Sciences of the Czech Republic, Boční II 1401, 14131 Prague 4, Czech Republic \\ ${ }^{3}$ ESA secondment, Space Telescope Science Institute, Baltimore, MD 21218, U.S.A.
}

Received December 16, 1996; accepted January 21, 1997

\begin{abstract}
We present a near-IR, mainly $H$ band, photometry of 72 nearby $(d<40 \mathrm{Mpc})$ disk galaxies. The main goal of the survey was to search for isophotal twist inside their nuclear regions. As the twist can be due in some cases to projection effects, rather than resulting from a dynamical phenomenon, we deproject - under the simplifying assumption of a 2D geometry - all galaxies whose disk position angle and inclination are known, the latter not exceeding $75^{\circ}$. We show the ellipticity, position angle and surface brightness radial profiles, and discuss how a projection of $2 \mathrm{D}$ and $3 \mathrm{D}$ bars can distort the isophotes, give an illusion of a non-existing double bar or mask a real one. We report 15 new double-barred galaxies and confirm 2 detected previously. We identify 14 additional twists not known before and we also find nuclear triaxial structures in three SA galaxies. The frequency of Seyferts among galaxies with nuclear bars or twists is high. Since these observations are part of a larger survey, the interpretation of the results will be given in a future paper, as soon as the number of objects grows enough to permit meaningful statistics. As a secondary product, we publish structural parameters (length and axis ratio) of large-scale bars in order to extend still scarce data on bars in the near-IR.
\end{abstract}

Key words: galaxies: photometry — galaxies: spiral — galaxies: fundamental parameters - infrared: galaxies

\section{Introduction}

Non-axisymmetric distortions (bars, ovals) in inner parts of disk galaxies are recognized, due to N-body simulations including gas (e.g. Friedli \& Benz 1993; Combes 1994), to be an efficient mechanism for driving the interstellar medium (ISM) into the nuclear region. Numerous observations complete the picture by showing that various

Send offprint requests to: B. Jungwiert ${ }^{1}$ kinds of central activity, like Seyfert nuclei (e.g. Hummel et al. 1987), LINERs and starbursts (e.g. Devereux 1989; Telesco et al. 1993) are often correlated with the presence of bars (note however counter-examples of many Seyferts (McLeod \& Rieke 1995), as well as galaxies with strong IR excess (Pompea \& Rieke 1990), that do not show any non-axisymmetric deviation).

While a large-scale bar is probably sufficient to fuel a starburst occuring inside a few hundred parsecs, it seems unable to bring the ISM down to the scale governed by a central blackhole $(<10 \mathrm{pc})$ that presumably powers active galactic nuclei (AGNs). The "bar-within-bar" scenario was proposed by Shlosman et al. (1989) to overcome the problem: first, a large-scale bar accumulates gas in a sub-kpc nuclear disk, that undergoes, when becoming massive enough, a secondary bar-forming instability, susceptible to funnel the ISM down to the $\mathrm{BH}$ region. The possibility to create such a double-bar, with the inner component rotating at a higher angular rate, was demonstrated in simulations of Friedli \& Martinet (1993).

In turn, searches for inner isophotal twists that would observationally confirm the existence of double-bar configurations were initiated. Preexisting detections of twists in spiral galaxies (de Vaucouleurs 1974; Kormendy 1979, 1982; Jarvis et al. 1988; Pompea \& Rieke 1990; Buta 1990; Buta \& Crocker 1993, BC93 hereafter) were substantially multiplied due to near-IR observations of Shaw et al. $(1993,1995)$ and $B V R I$ survey of Wozniak et al. (1995, W95 hereafter). Further twists in the near-IR were reported by Elmegreen et al. (1996, E96 hereafter) who summarized the preceding surveys: 51 isophote twists were discovered amongst 80 barred spirals and lenticulars (the frequency is insignificant since galaxies where the twist was expected were observed preferentially). The last authors also examined blue plates in the Sandage \& Bedke atlases and found 18 additional galaxies displaying the twist. Finally, Rauscher (1995) adds 5 other examples in the near-IR, raising the number of twist detections to 74 . 
A (considerable) part of twists can be due to projection effects on triaxial structures - bars, bulges or combination of both - with varying excentricity but no intrinsic (i.e. face-on viewed) variation of isophotal position angle.

For the intrinsic twists, a scenario competitive with the bar-within-bar hypothesis was suggested by Shaw et al. (1993) on the grounds of the orbital structure inside a bar: a gaseous ring between two inner Lindblad resonances (ILRs), phase-shifted with respect to the main bar due to its association with $x_{2}$ orbits, perturbs gravitationally the stellar component thus causing its isophote twist. In contrast to the double-bar of Friedli \& Martinet, the perturbed region can be tilted only towards the leading side of the main bar, both components rotating at the same pattern speed.

Recently, Davis \& Hunter (1995) and Friedli (1996) extended the panorama of double-bar dynamics by considering counter-rotating nuclear bars.

There are numerous open questions concerning the twists in disk galaxies: What is the frequency of the twist phenomenon? What is the fraction of intrinsic twists? How frequent are triaxial bulges? Is there a significant correlation between the intrinsic twists and the presence of nuclear activity? Are the intrinsic twists correlated with inner/nuclear rings? What mechanism is responsible for the intrinsic twists: bar-within-bar instability or gas perturbing stars between the ILRs, or both? How the twist properties vary along the Hubble sequence?

The above questions have no definitive answers mainly because of incompleteness of existing surveys, their bias towards galaxies with enhanced nuclear activity, insufficient resolution close to galactic centers where the twists occur, and projection effects.

This survey is intended to enlarge the set of disk galaxies showing the nuclear isophote twist and to quantify it for future statistical purposes. The paper is organized as follows: Sect. 2 summarizes observations and data reduction procedures including the ellipse fitting on isophotes. Section 3 deals, on a qualitative level, with projection and deprojection of bars and double bars, since this problem is crucial for establishing meaningful statistics and conclusions about the nature of twists. Individual galaxies are shortly described in Sect. 4, conclusions are outlined in Sect. 5. The contour plots as well as profiles of ellipticity, position angle and surface brightness along bars are given in the appendix.

\section{Observations and data reduction}

The data (Table 1) were acquired on three nights (February 27 to March 1, 1995) using the infrared camera IRAC2 installed on the 2.2-meter telescope at the ESO's La Silla Observatory. This camera is equipped with a Hg:Cd:Te NICMOS3 array of $256 \times 256$ pixels. The detector scale was chosen to be $0.52 \mathrm{arcsec} / \mathrm{pixel}$ corresponding to the field of view of about $2 \times 2$ arcminutes. The seeing on the first night was $1.2^{\prime \prime}$ ( $F W H M$ ) for all the three filters; during the second and the third nights it got reduced to $0.9^{\prime \prime}$ and $1.0^{\prime \prime}$ in the $H$-band (observations in bands $K$ and $J$ were carried out during the first night only).

Typically (but not always; see Table 1), four object frames were obtained for a galaxy in one band: exposure length for filters $H, J$ and $K$ was respectively $50 \mathrm{~s}$ (achieved by 5 elementary integrations of $10 \mathrm{~s}$ each, in order to avoid the detector saturation $), 30 \mathrm{~s}(1 \times 30 \mathrm{~s})$ and $50 \mathrm{~s}(10 \times 5 \mathrm{~s})$, resulting in the total integration time of $200 \mathrm{~s}, 120 \mathrm{~s}$ and $200 \mathrm{~s}$. To reduce the contamination by defective pixels (less than 1\%), the telescope pointing was shifted by a few arcseconds for every object frame.

Since the sky in the near-IR varies on the timescale of the total integration time, a sky frame (of the same exposure length as for an object frame) was taken after each object frame: the typical observing sequence was thus OBJECT-SKY-O-S-O-S-O-S. The sky frames were offset from a galaxy by a few arcminutes. Dark current frames of all relevant exposure times were prepared as well.

The data was reduced by means of the ESO MIDAS package. First, from each object frame the subsequent sky frame was subtracted (no dark subtraction was needed here because of equal exposure lengths). The resulting images were divided by the flatfield (normalized to unity) to eliminate the variation in the pixel-to-pixel response (about 10\%); the flatfield frame was constructed for each galaxy separately by median combining of dark-subtracted sky frames. In turn, the sky-subtracted and flatfielded images were aligned and averaged into one frame that was cleaned from remaining bad pixels (bi-linear interpolation) and intervening stars (bi-quadratic interpolation).

Table 1 summarizes basic information about observed objects: Column (1) Galaxy identification (N = NGC, $\mathrm{E}=\mathrm{ESO}, \mathrm{I}=\mathrm{IC})$, Column (2) Type according to RC3 (de Vaucouleurs et al. 1993), Column (3) Exposure time in filter $H$; four galaxies were observed also in $K$ : NGC $1433(4 \times 30 \mathrm{~s}), 3346(2 \times 50), 3887(4 \times 50), 5236$ $(4 \times 50)$, and five in $J:$ NGC 1433, 3384, 3593, 3887, 5236 ( $4 \times 30 \mathrm{~s}$ all), Column (4) $25 \mathrm{~B}$-mag/ $\operatorname{arcsec}^{2}$ isophotal diameter (from the Lyon-Meudon Extragalactic Database (LEDA), Paturel et al. 1989), Column (5) kinematical distance corrected for the Virgocentric inflow, $H_{0}=$ $75 \mathrm{~km} / \mathrm{s} / \mathrm{Mpc}$ (from LEDA), Column (6) Nuclear activity, rings, spirals: from (a) Véron-Cetty \& Véron (1996), (b) Telesco et al. (1993), (c) Devereux (1989), (d) Buta \& Crocker (1993).

\subsection{Calibration}

To calibrate images, three standard infrared stars were observed each night. The rms error in the determination of the photometric zero points was $0.03 \mathrm{mag}$ for all three filters on the first night. The zero points for the second and third night in band $H$ were consistent to within the error with that for the first night and all the three were aver- 
Table 1. Observed galaxies

\begin{tabular}{|c|c|c|c|c|c|}
\hline Galaxy & $\begin{array}{l}\text { Type } \\
\text { (RC3) }\end{array}$ & $\begin{array}{l}\tau_{H} \\
(\mathrm{~s})\end{array}$ & $\begin{array}{r}D_{25} \\
\left({ }^{\prime \prime}\right)\end{array}$ & $\begin{array}{r}d \\
{[\mathrm{Mpc}]}\end{array}$ & $\begin{array}{l}\text { Nuclear activity, } \\
\text { nucl. rings (nr), } \\
\text { nucl. spirals (ns) }\end{array}$ \\
\hline N 613 & $\mathrm{SB}(\mathrm{rs}) \mathrm{bc}$ & $3 \times 50$ & 380 & 17.9 & $\mathrm{Sey}^{a}, \mathrm{~ns}^{d}$ \\
\hline N 1079 & $\operatorname{RSAB}(\mathrm{rs}) 0 / \mathrm{a}$ & $4 \times 50$ & 208 & 17.1 & \\
\hline N 1187 & $\mathrm{SB}(\mathrm{r}) \mathrm{c}$ & $4 \times 50$ & 330 & 16.7 & \\
\hline N 1255 & $\mathrm{SAB}(\mathrm{rs}) \mathrm{bc}$ & $4 \times 50$ & 250 & 20.3 & \\
\hline N 1302 & $\operatorname{RSB}(\mathrm{r}) 0 / \mathrm{a}$ & $4 \times 50$ & 233 & 20.5 & $\mathrm{nr}^{d}$ \\
\hline N 1353 & $\mathrm{SB}(\mathrm{rs}) \mathrm{b}$ & $3 \times 50$ & 203 & 18.4 & \\
\hline N 1365 & $\mathrm{SB}(\mathrm{s}) \mathrm{b}$ & $2 \times 50$ & 673 & 19.4 & Sey $1 / \mathrm{H} \mathrm{II}^{a, b}, \mathrm{~ns}^{d}$ \\
\hline N 1398 & $\mathrm{R}^{\prime} \mathrm{SB}(\mathrm{r}) \mathrm{ab}$ & $2 \times 50$ & 425 & 16.5 & \\
\hline N 1433 & R'SB(r)ab & $4 \times 30$ & 387 & 11.1 & $\mathrm{nr}^{d}$ \\
\hline N 1512 & $\mathrm{SB}(\mathrm{r}) \mathrm{a}$ & $4 \times 50$ & 535 & 11.1 & $\mathrm{nr}^{d}$ \\
\hline N 1518 & $\mathrm{SB}(\mathrm{s}) \mathrm{dm}$ & $4 \times 50$ & 181 & & \\
\hline N 1640 & $\mathrm{SB}(\mathrm{r}) \mathrm{b}$ & $3 \times 50$ & 158 & 19.2 & \\
\hline N 1744 & $\mathrm{SB}(\mathrm{s}) \mathrm{d}$ & $4 \times 50$ & 488 & 7.4 & \\
\hline N 1784 & $\mathrm{SB}(\mathrm{r}) \mathrm{c}$ & $4 \times 50$ & 239 & 29.2 & \\
\hline N 1792 & $\mathrm{SA}(\mathrm{rs}) \mathrm{bc}$ & $4 \times 50$ & 315 & 13.2 & \\
\hline N 1808 & $\operatorname{RSAB}(\mathrm{rs}) \mathrm{a}$ & $3 \times 50$ & 387 & 10.4 & $\mathrm{H} \mathrm{II}^{a, b}, \mathrm{nr}^{, d}$ \\
\hline N 1832 & $\mathrm{SB}(\mathrm{r}) \mathrm{bc}$ & $4 \times 50$ & 154 & 24.0 & \\
\hline N 2217 & $\mathrm{RSB}(\mathrm{rs}) 0+$ & $4 \times 50$ & 268 & 19.0 & \\
\hline N 2442 & $\mathrm{SAB}(\mathrm{s}) \mathrm{bc}$ & $2 \times 50$ & 330 & 15.5 & \\
\hline N 2525 & $\mathrm{SB}(\mathrm{s}) \mathrm{c}$ & $4 \times 50$ & 173 & 19.3 & \\
\hline N 2811 & $\mathrm{SB}(\mathrm{rs}) \mathrm{a}$ & $4 \times 50$ & 151 & 31.6 & \\
\hline N 2911 & $\mathrm{SA}(\mathrm{s}) 0$ & $4 \times 50$ & 244 & 42.3 & Sey $3^{a}$ \\
\hline N 2935 & $\mathrm{R}^{\prime} \mathrm{SAB}(\mathrm{s}) \mathrm{b}$ & $4 \times 50$ & 218 & 28.3 & $\mathrm{nr}^{d}$ \\
\hline N 2997 & $\mathrm{SAB}(\mathrm{rs}) \mathrm{c}$ & $3 \times 50$ & 535 & 11.9 & $\mathrm{nr}^{d}$ \\
\hline N 3166 & $\mathrm{SAB}(\mathrm{rs}) 0 / \mathrm{a}$ & $4 \times 50$ & 287 & 17.4 & \\
\hline N 3346 & $\mathrm{SB}(\mathrm{rs}) \mathrm{cd}$ & $4 \times 50$ & 173 & 17.1 & \\
\hline N 3368 & $\mathrm{SAB}(\mathrm{rs}) \mathrm{ab}$ & $2 \times 150$ & 455 & 12.2 & \\
\hline N 3384 & SB(rs)0- & $4 \times 50$ & 330 & 10.1 & $?^{a}$ \\
\hline N 3393 & $\mathrm{R}^{\prime} \mathrm{SB}(\mathrm{rs}) \mathrm{a}$ & $4 \times 50$ & 131 & 47.6 & Sey $2^{a}$ \\
\hline N 3593 & $\mathrm{SA}(\mathrm{s}) 0 / \mathrm{a}$ & $4 \times 50$ & 315 & 8.7 & $n r^{d}$ \\
\hline N 3637 & $\mathrm{RSB}(\mathrm{r}) 0 / \mathrm{a}$ & $4 \times 50$ & 95 & 23.6 & \\
\hline N 3673 & $\mathrm{SB}(\mathrm{rs}) \mathrm{b}$ & $4 \times 50$ & 218 & 23.9 & \\
\hline N 3885 & $\mathrm{SA}(\mathrm{s}) 0 / \mathrm{a}$ & $4 \times 50$ & 144 & 22.1 & \\
\hline N 3887 & $\mathrm{SB}(\mathrm{r}) \mathrm{bc}$ & $4 \times 50$ & 199 & 14.8 & \\
\hline N 4050 & $\mathrm{SB}(\mathrm{r}) \mathrm{ab}$ & $4 \times 50$ & 185 & 23.6 & \\
\hline N 4106 & $\mathrm{SB}(\mathrm{s}) 0+$ & $4 \times 50$ & 97 & 26.8 & \\
\hline N 4178 & $\mathrm{SB}(\mathrm{rs}) \mathrm{dm}$ & $4 \times 50$ & 308 & & \\
\hline N 4192 & $\mathrm{SAB}(\mathrm{s}) \mathrm{ab}$ & $4 \times 50$ & 586 & & Sey $3^{a}$ \\
\hline N 4212 & SAc & $4 \times 50$ & 190 & & \\
\hline N 4216 & $\mathrm{SAB}(\mathrm{s}) \mathrm{b}$ & $4 \times 50$ & 488 & & \\
\hline N 4267 & $\mathrm{SB}(\mathrm{s}) 0-$ & $4 \times 50$ & 194 & 14.8 & \\
\hline N 4424 & $\mathrm{SB}(\mathrm{s}) \mathrm{a}$ & $4 \times 50$ & 218 & & \\
\hline N 4438 & $\mathrm{SA}(\mathrm{s}) 0 / \mathrm{a}$ & $4 \times 50$ & 511 & & Sey $3^{a}$ \\
\hline N 4442 & $\mathrm{SB}(\mathrm{s}) 0$ & $4 \times 50$ & 274 & 7.7 & \\
\hline N 4454 & $\mathrm{RSB}(\mathrm{r}) 0 / \mathrm{a}$ & $4 \times 50$ & 120 & 30.3 & \\
\hline N 4461 & $\mathrm{SB}(\mathrm{s}) 0+$ & $4 \times 50$ & 213 & 26.4 & \\
\hline N 4501 & $\mathrm{SA}(\mathrm{rs}) \mathrm{b}$ & $4 \times 50$ & 415 & 31.2 & Sey $2^{a}$ \\
\hline N 4503 & SB0- & $4 \times 50$ & 213 & 18.7 & \\
\hline N 4519 & $\mathrm{SB}(\mathrm{rs}) \mathrm{d}$ & $2 \times 50$ & 190 & 16.7 & \\
\hline N 4546 & $\mathrm{SB}(\mathrm{s}) 0-$ & $2 \times 50$ & 199 & 13.7 & \\
\hline N 4612 & $\mathrm{SB}(\mathrm{r}) 0+$ & $2 \times 50$ & 239 & 24.8 & \\
\hline N 4665 & $\mathrm{SB}(\mathrm{s}) 0 / \mathrm{a}$ & $4 \times 50$ & 228 & 10.6 & \\
\hline N 4684 & $\mathrm{SB}(\mathrm{r}) 0+$ & $3 \times 50$ & 173 & 20.8 & \\
\hline N 4689 & $\mathrm{SA}(\mathrm{rs}) \mathrm{bc}$ & $4 \times 50$ & 256 & 22.4 & \\
\hline N 4694 & SB0 & $3 \times 50$ & 190 & 16.4 & $\mathrm{H} \mathrm{II}^{a}$ \\
\hline N 4731 & $\mathrm{SB}(\mathrm{s}) \mathrm{cd}$ & $4 \times 50$ & 396 & 19.5 & \\
\hline N 4781 & $\mathrm{SB}(\mathrm{rs}) \mathrm{d}$ & $4 \times 50$ & 208 & 16.1 & \\
\hline N 4856 & $\mathrm{SB}(\mathrm{s}) 0 / \mathrm{a}$ & $3 \times 50$ & 256 & 17.0 & \\
\hline N 4900 & $\mathrm{SB}(\mathrm{rs}) \mathrm{c}$ & $3 \times 50$ & 134 & 13.1 & \\
\hline N 4902 & $\mathrm{SB}(\mathrm{r}) \mathrm{b}$ & $4 \times 50$ & 181 & 35.2 & \\
\hline N 4984 & $\mathrm{RSAB}(\mathrm{rs}) 0+$ & $4 \times 50$ & 165 & 15.2 & $?^{c}, \mathrm{nr}^{d}$ \\
\hline N 5101 & $\mathrm{RSB}(\mathrm{rs}) 0 / \mathrm{a}$ & $4 \times 50$ & 322 & 23.2 & \\
\hline N 5236 & $\mathrm{SAB}(\mathrm{s}) \mathrm{c}$ & $4 \times 50$ & 773 & & $\mathrm{H} \mathrm{II}{ }^{b}, \mathrm{nr}^{d}$ \\
\hline N 5427 & $\mathrm{SA}(\mathrm{s}) \mathrm{c}$ & $4 \times 50$ & 169 & 35.3 & Sey $2^{a}, \mathrm{nr}^{d}$ \\
\hline N 5566 & $\mathrm{SB}(\mathrm{r}) \mathrm{ab}$ & $4 \times 50$ & 396 & 20.8 & \\
\hline N 5643 & $\mathrm{SAB}(\mathrm{rs}) \mathrm{c}$ & $4 \times 50$ & 274 & 13.7 & Sey $2^{a}$ \\
\hline N 5701 & $\mathrm{RSB}(\mathrm{rs}) 0 / \mathrm{a}$ & $3 \times 50$ & 256 & 20.9 & \\
\hline N 6753 & $\mathrm{RSA}(\mathrm{r}) \mathrm{b}$ & $4 \times 50$ & 147 & 39.3 & $\mathrm{nr}^{d}$ \\
\hline N 6782 & $\operatorname{RSAB}(r) a$ & $4 \times 50$ & 131 & 48.5 & $\mathrm{nr}^{d}$ \\
\hline N 6810 & $\mathrm{SA}(\mathrm{s}) \mathrm{ab}$ & $1 \times 50$ & 190 & 23.5 & \\
\hline E437-67 & $\mathrm{R}^{\prime} \mathrm{SB}(\mathrm{r}) \mathrm{ab}$ & $4 \times 50$ & 123 & 39.4 & $\mathrm{nr}^{d}$ \\
\hline I 1953 & $\mathrm{SB}(\mathrm{rs}) \mathrm{d}$ & $3 \times 50$ & 165 & 22.9 & \\
\hline
\end{tabular}

aged to give the single zero point. The airmass correction was applied using the mean atmospheric extinction coefficients for the observing site: $a_{H}=0.06, a_{J}=0.08, a_{K}=$ $0.11 \mathrm{mag} /$ airmass; the airmass falls between 1 and 2.03 for our observations.

To test the photometric reliability, we have compared the results of our calibration to published photometry. In the $H$ band, our sample has nine galaxies in common (NGC 1302, 1398, 1433, 1808, 2217, 5566, 5701, 6753 and 6810) with the aperture photometry of Griersmith et al. (1982). We have simulated the apertures of diameter $22^{\prime \prime}$, $33^{\prime \prime}$ and $56^{\prime \prime}$ on our frames and found a mean magnitude difference, $\Delta m_{H}=m_{H \text {, ours }}-m_{H, \mathrm{G} 82}$, of $-0.19( \pm 0.11)$, $-0.14( \pm 0.10)$ and $-0.14( \pm 0.09)$. The $H$-band aperture photometry of 10 other galaxies of our survey (NGC 3166, 3885, 3887, 4212, 4273, 4501, 4781, 4900, 4902 and 4984) was done by Devereux (1989): our magnitudes for his $9.3^{\prime \prime}$ aperture differ by $\Delta m_{H}=-0.09( \pm 0.12)$. Both comparisons given above could indicate a systematic offset of our calibration by $0.1-0.2 \mathrm{mag}$, however this number is within the errors quoted in the referenced papers. Another galaxy (NGC 2997) was measured by Forbes et al. (1992): in this case $\Delta m_{H}=+0.05$ and +0.07 for the $6^{\prime \prime}$ and $12^{\prime \prime}$ apertures. Finally, the surface photometry of Héraudeau et al. (1996) has one common object with us, NGC 6810 , for which we find $\Delta m_{H}=+0.05( \pm 0.08)$ along the $60^{\prime \prime}$ major-axis profile.

\subsection{Ellipse fitting}

To follow the isophotal twist, we have used the ellipse fitting algorithm FIT/ELL3 (in the MIDAS context SURFPHOT), developed by Bender \& Möllenhoff (1987) for the study of the isophotal twist in elliptical galaxies (cf. Sect. 3). To parametrize the bars and double bars we use terms and quantities introduced by W95 to whom we refer the reader for details: typically, for a nearly face-on galaxy (projection effects are discussed in Sect. 3) with two bars, the ellipticity $(e=1-b / a$, where $a$ and $b$ are the ellipse semi-major and semi-minor axes) first grows to a first local maximum $e_{\max }^{\mathrm{s}}$ (at $a=l_{\max }^{\mathrm{s}}$ ) corresponding to the secondary (i.e. inner) bar, then falls to a minimum $e_{\min }^{\mathrm{s}}$ before climbing again to a primary bar maximum, $e_{\max }^{\mathrm{p}}$ (at $\left.a=l_{\max }^{\mathrm{p}}\right)$, after which it decreases towards the ellipticity of the disk, $e_{\text {disk }}$ (see Fig. 1 in W95). We define the sizes of the bars by $l_{\max }^{\mathrm{s}}$ and $l_{\mathrm{max}}^{\mathrm{p}}$. Position angles (measured from the North counterclockwise) of the bars and the disk are denoted $\mathrm{PA}^{\mathrm{s}}, \mathrm{PA}^{\mathrm{p}}$ and $\mathrm{PA}_{\text {disk }}$. When the $\mathrm{PA}$ changes along a bar, we define $\mathrm{PA}^{\mathrm{s}}$ and $\mathrm{PA}^{\mathrm{p}}$ to be the $\mathrm{PA}$ at $l_{\max }^{\mathrm{s}}$ and $l_{\max }^{\mathrm{p}}$, respectively. In agreement with W95 and E96, we shall classify the bar isophotes as twisted whenever the variation of the $\mathrm{PA}$ along a bar exceeds $10^{\circ}$.

In the appendix, we present for individual galaxies the PAs and ellipticities plotted against the semi-major axis of the fitted ellipses which is scaled logarithmically in order to better see inner regions. We do not comment on any 
feature inside $a=3^{\prime \prime}$ since the ellipse fitting on artificial bars of known shapes proved not to be reliable there due to the seeing and small number of pixels. However, we show the profiles down to $a=1^{\prime \prime}$ since they often display a continuity below $a=3^{\prime \prime}$ and might provide a reference for eventual future observations with higher resolution. The unreliable region, $a<3^{\prime \prime}$, is separated by a vertical dash-dot line in plots.

\section{Projection effects on isophotes: twists in ellipticals and bars}

It has been known for more than three decades that axes of isophotal contours in many elliptical galaxies rotate (e.g. Liller 1960; Bertola \& Galletta 1979; Nieto et al. 1992). Such twists can be explained either by intrinsic misalignement of isophotal surfaces (which are ellipsoidal in the first approximation) or by projection effects: in the latter case, the aligned ellipsoids must be triaxial and their eccentricity must vary with radius at the same time. Models (e.g. Madejsky \& Möllenhoff 1990) show that even a moderate triaxiality can produce a considerable twist if one looks at an elliptical galaxy under oblique view.

It is natural to expect the isophote twist due to projection effects also in the case of galactic bars since they are obviously triaxial and their eccentricity shows a radial variation (as seen in galaxies viewed face-on). In looking for a correlation between central activity and isophote rotation, one should separate the intrinsic twists, related to dynamics, from mere projection twists.

The solution of such a task is outside the scope of this paper. Nevertheless we would like to initiate the discussion on that topic by several simple illustrative examples of projection effects on artificially constructed single and double bars.

Figure 1a shows the ellipticity and PA profiles of a faceon viewed $2 \mathrm{D}$ bar, whose isodensity contours are perfect ellipses with axial ratio $a / b$ varying radially from 1 to 3 . After projecting (with only moderate inclination, $I=30^{\circ}$ ) about the line with $\mathrm{PA}=\mathrm{PA}_{\text {proj }}=0^{\circ}$ (i.e. coinciding with the bar minor axis), the PA along the bar becomes twofold, with two plateaus separated by a sharp $90^{\circ}$-transition at which the ellipticity falls locally to zero (Fig. 1b). With the same $I$ but $\mathrm{PA}_{\text {proj }}=30^{\circ}$, one obtains a gradual twist of $\sim 50^{\circ}$ (Fig. 1c).

A $2 \mathrm{D}$ double barred system with the inner component perpendicular to the outer one is presented in Fig. 2a: the large-scale bar is the same as in the above case; the small one is 7 times shorter and its axial ratio $a / b$ varies linearly from 1 to 2 . The projection with $I=60^{\circ}$ and $\mathrm{PA}_{\text {proj }}=$ $60^{\circ}$ is shown in Fig. 2b: the local ellipticity maximum corresponding to the secondary bar nearly disappears; the PA varies along both primary and secondary bars.

Finally, a system of two parallel bars (with the same parameters as above) is shown in Fig. 3a. The projection by $I=60^{\circ}$ about the minor axis of both bars (i.e.
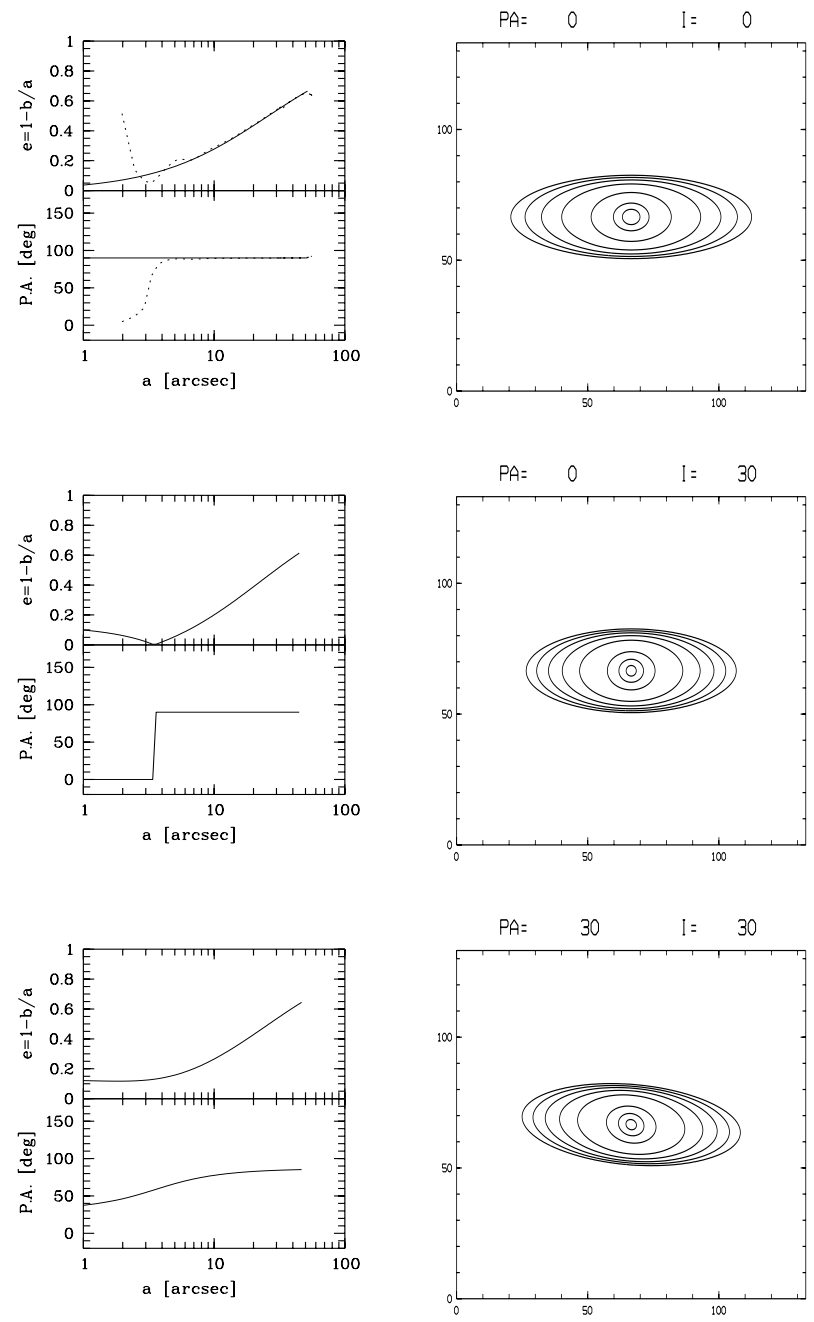

Fig. 1. Ellipticities $(1-b / a)$, position angles (PA) and contour plots for a single 2D bar: a) Face-on view - full lines in plots of radial profiles (dotted lines indicate the same quantities measured after the bar is first analytically projected and then numerically deprojected back; see the text), b) Projection with $I=30^{\circ}$ and $\mathrm{PA}_{\text {proj }}=0^{\circ}$, c) Projection with $I=30^{\circ}$ and $\mathrm{PA}_{\text {proj }}=30^{\circ}$

$\left.\mathrm{PA}_{\text {proj }}=0^{\circ}\right)$ results in an illusion of two perpendicular bars (Fig. 3b).

The above examples clearly demonstrate that the projection is a crucial factor for classifying twists and double bars. To disentangle projection effects from intrinsic distortions, one can try to deproject the observed images, making use of two advantages spiral galaxies have with respect to ellipticals: a) they are fairly two-dimensional except the bulge region and b) the inclination $I$ and position angle PA can be deduced from the shape of the outer disk under the assumption that it is intrinsically circular. A two-dimensional body with known $I$ and PA can be deprojected without ambiguity: if conditions a) and b) were strictly met, the problem would be solved. 

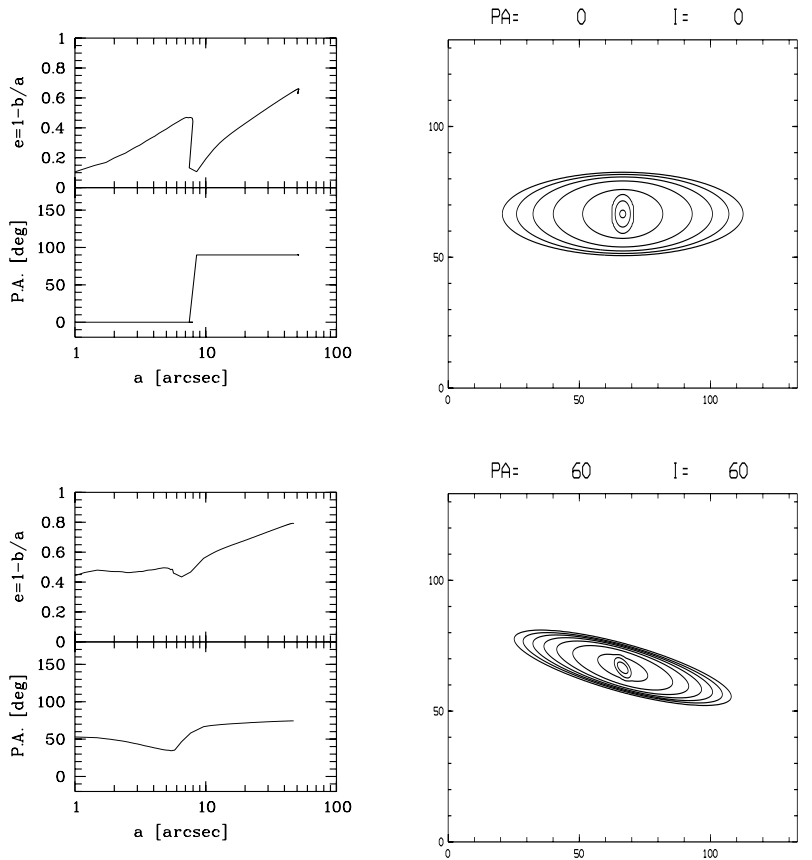

Fig. 2. Ellipticities $(1-b / a)$, position angles $(\mathrm{PA})$ and contour plots for a a 2D double bar - bars perpendicular: a) Face-on view, b) Projection with $I=60^{\circ}$ and $\mathrm{PA}_{\text {proj }}=60^{\circ}$

Nevertheless many complications exist: the bulge is clearly three-dimensional; the primary bar may also be significantly thickened close to the center due to the scattering on vertical resonances (e.g. Combes et al. 1990); the secondary bar, when it exists, is confined to that bulge-bar $3 \mathrm{D}$ region; the outer disk has not necessarily the intrinsic circular shape which can result in substantial errors in determining $I$ and PA.

In this paper, we have deprojected, under the assumption of two-dimensionality, galaxies with inclination lower than $75^{\circ}$, and we present, in the appendix, the deprojected radial profiles together with the projected ones. Since outer disks are usually located outside our images, we have used disk inclination and position angles quoted in the Lyon-Meudon Extragalactic Database (LEDA, Paturel et al. 1989).

The deprojection can be done in two ways: either the image is first deprojected and then a new ellipse fitting is carried out or the ellipses fitted to the projected image are deprojected analytically. The two approaches are not completely equivalent because of discreteness of the detector array and because the isophotes are not perfect ellipses. Our experiments have shown that the first method is less reliable: after deprojecting the image, one has to interpolate to get intensity at pixel positions which causes numerical errors resulting in spurious variations of ellipticity and position angle in the subsequent ellipse fitting. We show this effect by dotted curves in Fig. 1a: they correspond to the bar which is first projected with $I=30^{\circ}$
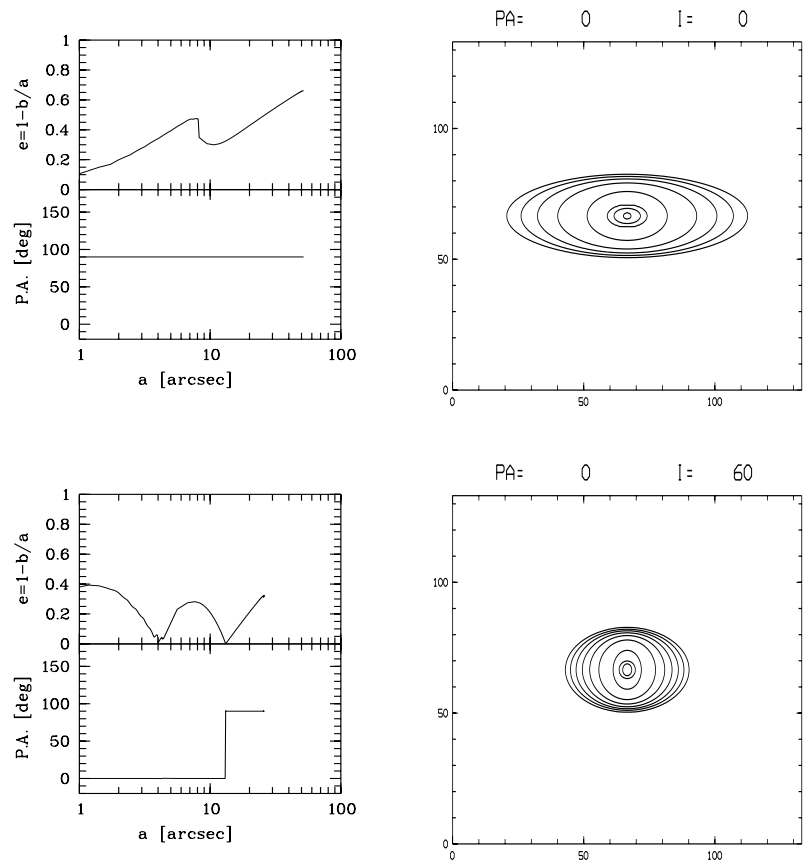

Fig. 3. Ellipticities $(1-b / a)$, position angles (PA) and contour plots for a a 2D double bar - bars parallel: a) Face-on view, b) Projection with $I=60^{\circ}$ and $\mathrm{PA}_{\text {proj }}=0^{\circ}$,

and $\mathrm{PA}_{\text {proj }}=30^{\circ}$ (Fig. 1c) and then deprojected back to the face-on position. Both ellipticity and position angle profiles significantly differ from the correct ones (full lines) inside $a \sim 6^{\prime \prime}$, possibly giving illusion of a small secondary bar. This numerical error is expected to occur in regions with high density contrasts, e.g. close to the center or at the edges of bars. Therefore we have preferred the second approach.

Whether the deprojected profiles are meaningful or not, depends on how closely individual galaxies fulfil the conditions a) and b) given above. Clearly, in regions with non-negligible thickness, the error resulting from the deprojection will grow with the galaxy inclination. As can be seen from the profiles of observed galaxies, the deprojection does not look reasonable in the bulge region if $I$ exceeds $\sim 45^{\circ}$. Additional error is introduced by uncertainties in $I$ and $\mathrm{PA}^{\text {disk }}$.

Being aware of big uncertainties in the deprojection procedure, we do not rely on it to draw firm conclusions about the nature of a twist but use it only as a secondary help: if a double bar (or gradual twist) seen on the projected image remains after deprojection, we consider the probability of its existence to be strengthened; if it disappears, while $I<45^{\circ}$, we take it to be a projection effect; on the other hand if a double bar structure appears only after deprojection, we do not classify it to be a double bar. 


\section{Individual galaxies}

\subsection{Two triaxial structures (double bars)}

We include into this section all galaxies which have, or at least are suspected to have, more than one triaxial structure. We do not strictly distinguish between double bars and bars with embedded triaxial bulges since this difference is rather elusive. Usually, we speak about double bars; only in cases when the inner ellipticity maximum is low (after deprojection) and/or not clearly separated from the outer one we use the latter term. The classification of some galaxies as double-barred is speculative, because either they are too inclined or the central resolution is low. NGC 613 (SBbc/Seyfert, $1^{\prime \prime} \sim 86 \mathrm{pc}, I=35.1^{\circ}$ ):

This Seyfert galaxy is known to possess, inside the largescale bar, an optical nuclear spiral. BC93 give semi-major axes of the feature $7 \times 6^{\prime \prime}$. We identify short nuclear spiral arms also in the near-IR and suggest, on the basis of both the ellipse fitting and the inspection of the grey-scale image, that they have an associated nuclear bar $\left(e_{\max }^{\mathrm{s}}=0.55\right.$ at $\left.a=5.3^{\prime \prime}\right)$. If our interpretation is correct, then NGC 613 is a new example of a double-barred system. The primary bar peaks $\left(e_{\max }^{\mathrm{p}}>0.72\right)$ outside our image.

NGC $1079\left(\mathrm{RSAB} 0 / \mathrm{a}, 1^{\prime \prime} \sim 82 \mathrm{pc}, I=57.1^{\circ}\right)$ :

The highest peak in ellipticity $\left(e_{\max }=0.51\right.$ at $\left.a \sim 32^{\prime \prime}\right)$ is related to the large-scale bar, however the shape of the profile can be deformed by the presence of spiral arms at the end of the bar. There is a secondary maximum $\left(e_{\max }=\right.$ $0.32^{\mathrm{s}}$ at $\left.a=17^{\prime \prime}\right)$ that we attribute to the triaxiality of the bulge.

NGC $1353\left(\mathrm{SBb}, 1^{\prime \prime} \sim 86 \mathrm{pc}, I=70.2^{\circ}\right)$ :

The galaxy inclination is rather high and the interpretation therefore uncertain: we suggest that the innermost ellipticity peak is associated with a nuclear bar along which the PA varies as a result of projection effects. The largescale bar is reflected by the PA plateau around $a=14^{\prime \prime}$ and a corresponding small bump in the ellipticity. The outermost ellipticity maximum is related to large-scale spiral arms. The deprojection, although done, is considered to be unreliable.

NGC $1365\left(\mathrm{SBb} /\right.$ Seyfert $\left.1,1^{\prime \prime} \sim 93 \mathrm{pc}, I=58.1^{\circ}\right)$ :

The galaxy has an IR-bright Seyfert nucleus and, as in the case of NGC 613, a nuclear spiral, best seen as prominent dust lanes in optical images (e.g. Teuben et al. 1986). NGC 1365 is also classified as a starburst galaxy (e.g. Telesco et al. 1993), with the star-forming activity concentrated in circumnuclear "hot-spots".

The morphology of the nuclear region is complex and patchy also in our $H$ image, indicating that the emission of old red stars is probably strongly contaminated by the light of new red giants and supergiants formed in the starburst. The nuclear spiral is well recognized and we identify a nuclear bar embedded in it: the peak in the ellipticity $\left(e_{\max }^{\mathrm{s}}=0.46\right.$ at $\left.a=8.3\right)$ is related to that bar while the adjacent minimum in the PA is related to the nuclear spiral. The nuclear bar is roughly parallel (NE - SW) to the elliptical distribution of circumnuclear molecular gas mapped by Sandqvist et al. (1995). Neither the spiral nor the bar are smooth, unlike in NGC 613.

NGC 1398 (R'SBab, $1^{\prime \prime} \sim 80 \mathrm{pc}, I=48.0^{\circ}$ ):

The disk is dominated by a well defined large-scale bar reaching $e_{\max }(0.37)$ at $a=36^{\prime \prime}$ after which it passes into an outer ring. The inner isophotes $\left(a<20^{\prime \prime}, \mathrm{PA} \sim 80-\right.$ $\left.90^{\circ}\right)$ are not aligned with the primary bar $\left(\mathrm{PA}^{\mathrm{p}} \sim 12^{\circ}\right)$ and are slightly twisted (by $\sim 13^{\circ}$ between 5 and $12^{\prime \prime}$ ). A small bump in profiles near $a=14^{\prime \prime}$ was found also by W95 but they were reluctant to interpret it. We consider it is related to the triaxiality of the bulge; its signature is seen also in deprojected profiles $\left(\mathrm{PA}_{\text {disk }}=100^{\circ}\right)$. Note that the inner isophote twist almost disappears after deprojection: the $\mathrm{PA}$ is constant (within $7^{\circ}$ ) along the whole $60^{\prime \prime}$-profile.

NGC 1433 (R'SBab, $1^{\prime \prime} \sim 53 \mathrm{pc}, I=24.5^{\circ}$ ):

The double barred structure of the galaxy was already reported by Buta (1986) and W95 in BVRI bands. We find, in $J H K$ bands, the length $l_{\max }^{\mathrm{s}}$ of the secondary bar to be 5.6, 5.4 and $6.2^{\prime \prime}$. For comparison with W95 we measured also $l_{\text {min }}^{\mathrm{s}}=12.9,13.0$ and $12.8^{\prime \prime}$ (W95 give $11.5^{\prime \prime}$ in filter $I$ ). The primary bar exceeds in length our frame, so that its ellipticity is still increasing at the last point of the profile. The PAs of bars are 32 and $95^{\circ}$ (with the nuclear bar leading), in good agreement with W95 (30 and $94^{\circ}$ ).

NGC $1512\left(\mathrm{SBa}, 1^{\prime \prime} \sim 43 \mathrm{pc}, I=62.9^{\circ}\right)$ :

The image is dominated by a large-scale bar showing isophotal twist $\left(\triangle \mathrm{PA} \sim 30^{\circ}\right)$ exterior to $a \sim 7^{\prime \prime}$ (noticed by E96 on the blue plate). A low ellipticity maximum and the associated change of the PA at $a \sim 6^{\prime \prime}$ can be indicative of a distinct component, probably a triaxial bulge. This interpretation is however uncertain because of high inclination (note a partial similarity with Fig. 2 showing a projection of a double barred system).

NGC $1808\left(\mathrm{RSABa} / \mathrm{HII}, 1^{\prime \prime} \sim 50 \mathrm{pc}, I=60.6^{\circ}\right)$ :

NGC 1808 is a nearby starburst galaxy (e.g. Telesco 1993) with many star forming knots inside $1 \mathrm{kpc}$. It is also suspected to possess a hidden Seyfert nucleus (e.g. VéronCetty \& Véron 1985).

On the $H$ image the nuclear region looks smooth, unlike in optical. This is consistent with Tacconi-Garman et al. (1996) who claim that the near-IR emission from the nucleus of this galaxy is dominated by old stars with only a small $(<10 \%)$ contribution from young red giants and supergiants born in the starburst. We interpret the peak in the eccentricity and the associated PA plateau at $a \sim 3^{\prime \prime}$ as a manifestation of a nuclear bar (its presence was recently deduced also by Kotilainen et al. (1996) on the basis of $J H K$ contour plots). We do not attempt to give parameters of the large-scale bar since the galaxy has a peculiar morphology (possibly due to the interaction with NGC 1792) and our image is spatially rather small. Phillips (1993) classifies the galaxy as barred on the basis of the distribution of HII regions and Saikia et al. 
(1990) report an HI bar $22 \mathrm{kpc}$ long. Therefore we classify NGC 1808 as being double-barred.

NGC $2217\left(\mathrm{RSB} 0+, 1^{\prime \prime} \sim 91 \mathrm{pc}, I=28.6^{\circ}\right)$ :

We identify this low-inclination galaxy as a new doublebarred system: the two maxima in ellipticity $\left(e_{\max }^{\mathrm{s}}=0.19\right.$ at $a=7.8^{\prime \prime}$ and $e_{\max }^{\mathrm{p}}=0.48$ at $a=37^{\prime \prime}$ occur on the approximate plateaus of the $\mathrm{PA}\left(138^{\circ}\right.$ and $112^{\circ}$ for the secondary and primary bar, respectively).

NGC 2935 (R'SABb, $\left.1^{\prime \prime} \sim 136 \mathrm{pc}, I=43.4^{\circ}\right)$ :

The outer ellipticity peak corresponds to the large-scale bar $\left(e_{\max }=0.52\right.$ at $\left.a=25^{\prime \prime}\right)$. We interpret the secondary maximum, still perceptible after deprojection, as being related to another triaxial component, probably the bulge (the shape of the profile in the transition region between the two peaks can be partly deformed by the presence of a badly masked star near the major axis of the inner component at $a \sim 11^{\prime \prime}$, however the inspection of the grey-scale image confirms that the inner misaligned structure really exists). The smooth twist of the barred isophotes (already noticed by E96 on blue plates) is explainable by projection effects. After deprojecting, the PA is two-fold with two approximate plateaus.

NGC 3368 (SABab, $\left.1^{\prime \prime} \sim 59 \mathrm{pc}, I=51.0^{\circ}\right)$ :

One can see three ellipticity maxima $\left(e_{\max }=0.29,0.30\right.$ and 0.43 ) accompanied by three plateaus in the PA profile. The third maximum is controversial since it is close to the locus where spiral arms start and our frame ends at the same time. The illusion of the triple-barred system disappears after deprojection $\left(\mathrm{PA}_{\text {disk }}=5.0^{\circ}\right)$ : it seems plausible that the galaxy is double-barred with the two bars roughly aligned. However, as $I$ is rather high for the deprojection to be unambiguous, we do not exclude that three triaxial components coexist.

NGC $3393\left(\mathrm{R}^{\prime}\right.$ SBa/Sey 2, $\left.1^{\prime \prime} \sim 230 \mathrm{pc}, I=23.7^{\circ}\right)$ :

Inside the large-scale bar $\left(e_{\max }=0.46\right.$ at $\left.a=13.3\right)$, there is an isophote twist of $\sim 11^{\circ}$ between $a=3^{\prime \prime}$ and $4.4^{\prime \prime}$. We speculate that it could result from the existence of a small secondary bar, since there is a local maximum of ellipticity near $a=2^{\prime \prime}$, while the PA has a plateau there. The proximity of that region to the center makes the double-bar classification uncertain; higher resolution is needed to confirm the discovery.

NGC $4984\left(\mathrm{RSAB} 0+, 1^{\prime \prime} \sim 73 \mathrm{pc}, I=45.9^{\circ}\right)$ :

The ellipticity peak $\left(e_{\max }=0.23\right)$ around $a=4^{\prime \prime}$ is associated with a short plateau in the PA $\left(64^{\circ}\right.$ between 2.9 and $\left.4.1^{\prime \prime}\right)$. Since the peak is even better seen after deprojection $\left(\mathrm{PA}_{\mathrm{disk}}=15^{\circ}\right)$, we believe that it indicates the presence of a short bar. The outer ellipticity maximum $\left(e_{\max }=0.30\right.$ at $\left.a=30^{\prime \prime}\right)$ is related to the primary bar. Both bars are intrinsically nearly parallel provided the deprojection is correct (cf. Fig. 3).

NGC 5101 (RSB0/a, $\left.1^{\prime \prime} \sim 110 \mathrm{pc}, I=33.6^{\circ}\right)$ :

The isophotes inside the large-scale bar $\left(e_{\max }=0.56\right.$ at $\left.a=50^{\prime \prime}\right)$ are strongly twisted $\left(\Delta \mathrm{PA} \sim 106^{\circ}\right)$. Such a twist is not explainable by mere projection effects in view of rather low galaxy inclination. Either there is an intrinsic gradual twist or a nuclear bar as would suggest the ellipticity maximum and the PA plateau at $a<3^{\prime \prime}$ : however the proximity of this feature to the center as well as its low ellipticity $\left(e_{\max }=0.06\right.$ at $\left.a=2^{\prime \prime}\right)$ make this hypothesis very speculative.

NGC 5566 (SBab, $1^{\prime \prime} \sim 100$ pc, $\left.I=79.5\right)$ :

In spite of the high inclination of the galaxy, we interpret the profiles in terms of a double barred structure with the higher maximum in ellipticity $\left(e_{\max }^{\mathrm{s}}=0.56\right.$ at $a \sim 6^{\prime \prime}$ ) corresponding to the nuclear bar. The largescale bar has, due to projection, only a low ellipticity peak $\left(e_{\max }^{\mathrm{p}}=0.24\right.$ at $\left.a \sim 24^{\prime \prime}\right)$ and a short PA dip. We think that the deprojected profiles are not meaningful - because $I \sim 80^{\circ}-$ and show them only as illustration of how weak the deprojection procedure is for such high inclinations (e.g. note the constancy of the deprojected ellipticity at a high value near the center).

It is interesting to compare this galaxy with NGC 3166 since both are highly inclined and their morphology is apparently similar on undeprojected images: at first sight, both seem to show a double barred structure with the components roughly perpendicular. Nevertheless, in the light of Sect. 3 and Figs. 1 and 3, we classify NGC 3166 as having only a large-scale bar while NGC 5566 is claimed to have two bars. To grasp the difference, note that in the (undeprojected) ellipticity profile of NGC 3166, there is no local maximum interior to that of the large bar; rather, the ellipticity monotonically climbs towards the center. This is characteristic of projection effects: the behaviour is qualitatively similar to Fig. $1 \mathrm{~b}$ in which the bar is projected along its minor axis as is the case of NGC 3166 (in comparison, keep in mind that the profile of NGC 3166 reflects also the disk - which adds the change of ellipticity and PA rightwards from the bar ellipticity maximum - and that the inclinations are different - which changes the relative height of the peak). On the other hand, NGC 5566 shows two ellipticity peaks (excluding the outer raising slope related to the disk); ellipticity decreases towards the center. That is why we interpret the structure as double barred: no projection of a single bar with reasonable ellipticity profile (monotonically increasing as suggested by face-on single barred galaxies) can generate a secondary ellipticity peak.

NGC $6782\left(\mathrm{RSABa}, 1^{\prime \prime} \sim 230 \mathrm{pc}, I=44.4^{\circ}\right)$ :

A double bar structure, discussed already by BC93 and W95, exists, with the primary $\left(e_{\max }^{\mathrm{p}}=0.51, l_{\max }^{\mathrm{p}}=26^{\prime \prime}\right)$ and secondary $\left(e_{\max }^{\mathrm{s}}=0.36, l_{\max }^{\mathrm{s}}=3.4^{\prime \prime}\right)$ bars oriented at $\mathrm{PA}=178^{\circ}$ and $150^{\circ}$, respectively. The deprojection $\left(\mathrm{PA}_{\text {disk }}=45^{\circ}\right)$ does not alter this picture: two peaks in ellipticity, along which the PA is roughly constant, still exist.

ESO 437-67 (R'SBab, $\left.1^{\prime \prime} \sim 190 \mathrm{pc}, I=29.9^{\circ}\right)$ :

This galaxy could be double-barred since two peaks in ellipticity $\left(e_{\max }=0.23\right.$ at $2.5^{\prime \prime}$ and 0.62 at $\left.31.8^{\prime \prime}\right)$ are associated with approximate plateaus in the PA (139 and $\left.119^{\circ}\right)$. Nevertheless, this classification is speculative because the 
inner feature is rather close to the center: higher resolution is desirable to confirm the hypothesis.

Table 2. Projected parameters of double bars

$\begin{array}{lrrrrrr}\text { Galaxy } & e_{\max }^{\mathrm{s}} & \begin{array}{r}l_{\max }^{\mathrm{s}} \\ \left(^{\prime \prime}\right)\end{array} & \begin{array}{r}\mathrm{PA}^{\mathrm{s}} \\ \left(^{\circ}\right)\end{array} & e_{\max }^{\mathrm{p}} & \begin{array}{r}l_{\max }^{\mathrm{p}} \\ \left(^{\prime \prime}\right)\end{array} & \begin{array}{r}\mathrm{PA}^{\mathrm{p}} \\ \left(^{\circ}\right)\end{array} \\ \text { N 613 } & & & & & & \\ \text { N 1079 } & 0.55 & 5.3 & 122 & >0.72 & >59 & 127 \\ \text { N 1365 } & 0.46 & 8.2 & 46 & ? & ? & 122 \\ \text { N 1353 } & 0.40 & 3.6 & 131 & 0.31 & 14 & 184 \\ \text { N 1398 } & 0.14 & 14 & 83 & 0.37 & 36 & 12 \\ \text { N 1433(H) } & 0.37 & 5.4 & 32 & ? & ? & ? \\ \text { N 1433(J) } & 0.35 & 5.6 & 32 & ? & ? & ? \\ \text { N 1433(K) } & 0.36 & 6.2 & 32 & ? & ? & ? \\ \text { N 1512 } & 0.13 & 5.7 & 74 & 0.61 ? & 68 ? & 45 \\ \text { N 1808 } & 0.53 & 3.3 & 158 & ? & ? & ? \\ \text { N 2217 } & 0.19 & 7.8 & 138 & 0.47 & 37 & 112 \\ \text { N 2935 } & 0.32 & 10 & 153 & 0.52 & 25 & 134 \\ \text { N 3368 } & 0.29 & 4.2 & 130 & 0.30 & 24 & 156 \\ \text { N 3393 } & 0.20 & 2.0 & 147 & 0.46 & 13 & 159 \\ \text { N 4984 } & 0.23 & 4.0 & 64 & 0.30 & 30 & 95 \\ \text { N 5101 } & 0.06 & 2.0 & 46 & 0.56 & 50 & 121 \\ \text { N 5566 } & 0.56 & 5.9 & 37 & 0.24 & 24 & 156 \\ \text { N 6782 } & 0.36 & 3.4 & 150 & 0.51 & 26 & 178 \\ \text { E 437-67 } & 0.23 & 2.5 & 138 & 0.62 & 32 & 119\end{array}$

\subsection{SA galaxies with a nuclear bar}

NGC $1792\left(\mathrm{SAbc}, 1^{\prime \prime} \sim 64 \mathrm{pc}, I=62.0^{\circ}\right)$ :

The ellipticity maximum inside $10^{\prime \prime}$ is related to a short nuclear bar $\left(e_{\max }=0.45\right.$ at $\left.5^{\prime \prime}\right)$ along which no clear twist occurs. Further ellipticity maxima are connected to spiral arms.

NGC $4438\left(\mathrm{SA} 0 / \mathrm{a}(\mathrm{pec}) / \mathrm{Sey} 3, I=78^{\circ}\right)$ :

This galaxy (Arp 120), possessing a Seyfert nucleus, is interacting with a close companion NGC 4435 in the Virgo cluster Its nuclear regions might be perturbed by infalling gas (e.g. Combes et al. 1988; Kenney et al. 1995). Its inner maximum in ellipticity could reflect either a tidally deformed bulge or a nuclear bar.

NGC 5427 (SAc/Seyfert 2, $1^{\prime \prime} \sim 170$ pc, $\left.I=38.9^{\circ}\right)$ :

The ellipticity profile shows four clear maxima, the highest of which (at $a \sim 21^{\prime \prime}$ ) corresponds to the two-arm spiral structure. The two innermost ones $\left(a \sim 5^{\prime \prime}\right.$ and $\left.8^{\prime \prime}\right)$ do not correspond to well defined plateaus in PA, however the changes in ellipticity and PA occur at roughly the same places. We agree with W95 that these features are oval structures whose nature remains controversial, which is perhaps connected to the interacting nature of NGC 5427. Nevertheless, we note that after deprojection, the PA is defined much better in the inner region: it is constant up to $6^{\prime \prime}$, i.e. within the first ellipticity peak which could indicate a nuclear bar in this region. This interpretation is tempting also because of the Seyfert nucleus.

\subsection{Twisted bar isophotes}

In this section, galaxies with gradual twist along their large-scale bars are described. The selection is done on the basis of undeprojected images: some of such twists are likely not to be intrinsic.

NGC $1187\left(\mathrm{SBc}, 1^{\prime \prime} \sim 82 \mathrm{pc}, I=46.7^{\circ}\right)$ :

The ellipticity grows to its maximum $\left(e_{\max }=0.60\right)$ at $a=32^{\prime \prime}$, reflecting the large-scale bar from the tips of which two-arm spiral structure emanates. The PA is roughly constant inside the bar except the innermost region $\left(a=2-7^{\prime \prime}\right)$ where a $15^{\circ}$-twist occurs. After the deprojection $\left(\mathrm{PA}_{\text {disk }}=130^{\circ}\right)$, a possibility that the system is double-barred emerges: the PA is essentially two-fold and there is a new maximum in ellipticity at about $3^{\prime \prime}$. In case that the secondary bar really existed, it would be nearly perpendicular to the primary one $\left(\Delta \mathrm{PA}=87^{\circ}\right)$. However, we believe that such an appearance is likely to be an artefact of a wrong deprojection (cf. Fig. 1) and, in accordance with rules established in Sect. 3, we classify the galaxy only as having a twist.

NGC $1302\left(\mathrm{RSB} 0 / \mathrm{a}, 1^{\prime \prime} \sim 96 \mathrm{pc}, I=27.4^{\circ}\right)$ :

Between $a=3-7^{\prime \prime}$ the PA is roughly constant $\left(180-190^{\circ}\right)$ but then it turns by $20^{\circ}$ along the bar whose $e_{\max }(0.35)$ occurs at $a=29^{\prime \prime}$. This twist was already noted by W95 who attributed it to the presence of dust lanes since its amplitude depends on the band $(B V R I)$. It is important that we found it also in band $H$ where the dust extinction is much less important.

NGC 1832 (SBbc, $\left.1^{\prime \prime} \sim 115 \mathrm{pc}, I=46.3^{\circ}\right)$ :

The eccentricity of the large-scale bar grows continuously to $e_{\max }=0.58$ at $a=17^{\prime \prime}$ where a regular two-armed spiral structure starts. There is a twist of amplitude $14^{\circ}$ inside the bar. The twist is even more pronounced $(\triangle \mathrm{PA}=$ $\left.53^{\circ}\right)$ after deprojection $\left(\mathrm{PA}_{\text {disk }}=10^{\circ}\right)$.

NGC $2442\left(\mathrm{SABbc}, 1^{\prime \prime} \sim 74 \mathrm{pc}, I=27.8^{\circ}\right)$ :

The inner isophotes are twisted inside the large-scale bar: $\triangle \mathrm{PA} \sim 29^{\circ}$ between $a=3^{\prime \prime}$ and $10^{\prime \prime}$.

NGC $2525\left(\mathrm{SBc}, 1^{\prime \prime} \sim 93 \mathrm{pc}, I=48.2^{\circ}\right)$ :

The large-scale bar, with maximum ellipticity of 0.69 (at $\left.a=22^{\prime \prime}\right)$, has twisted isophotes: $\triangle \mathrm{PA}=13^{\circ}$. The deprojection $\left(\mathrm{PA}_{\text {disk }}=75^{\circ}\right)$ is suspicious: the resulting big twist is probably the consequence of unexact deprojection parameters and noisy image.

NGC 2997 (SABc, $\left.1^{\prime \prime} \sim 57 \mathrm{pc}, I=44.5^{\circ}\right)$ :

The twist of amplitude $25^{\circ}$ between $a=4^{\prime \prime}$ and $7.3^{\prime \prime}$ can be due to projection effects alone.

NGC $3166\left(\mathrm{SAB} 0 / \mathrm{a}, 1^{\prime \prime} \sim 84 \mathrm{pc}, I=73^{\circ}\right)$ :

Looking at inner contours, one could easily get impression that the galaxy is double-barred, with the bars roughly perpendicular one to another. However, this appearance is probably entirely due to projection effects (note that no ellipticity peak corresponds to the first PA plateau; cf. Fig. 1 and comments on NGC 5566). After deprojection $\left(\mathrm{PA}_{\text {disk }}=87^{\circ}\right)$, no clear isophote twist is found. 
NGC $3637\left(\mathrm{RSB} 0 / \mathrm{a}, 1^{\prime \prime} \sim 115 \mathrm{pc}, I=30.3^{\circ}\right)$ :

Inside the large-scale bar $\left(e_{\max }=0.38\right.$ at $a=14.6^{\prime \prime} ; \mathrm{PA}=$ $37^{\circ}$ ), a strong twist (amplitude $\sim 71^{\circ}$ in the region $a=$ $\left.3^{\prime \prime}-14.6^{\prime \prime}\right)$ is measured. The resolution in the inner part is not sufficient to say whether a secondary bar exists.

NGC $3673\left(\mathrm{SBb}, 1^{\prime \prime} \sim 115 \mathrm{pc}, I=51.6^{\circ}\right)$ :

There is a small twist ( $17^{\circ}$ between $a=3^{\prime \prime}$ and $\left.42^{\prime \prime}\right)$ inside the large-scale bar $\left(e_{\max }=0.73\right.$ at $\left.a=42^{\prime \prime}\right)$

NGC 3887 (SBbc, $\left.1^{\prime \prime} \sim 71 \mathrm{pc}, I=39.5^{\circ}\right)$ :

Inner isophotes (between $a=3^{\prime \prime}$ and $25^{\prime \prime}$ ) inside the largescale bar $\left(e_{\max }=0.68\right.$ at $\left.a=33^{\prime \prime} ; \mathrm{PA}=181^{\circ}\right)$ are twisted by $22^{\circ}$.

NGC $4454\left(\mathrm{RSB} 0 / \mathrm{a}, 1^{\prime \prime} \sim 140 \mathrm{pc}, I=29.5^{\circ}\right)$ :

There is a strong twist $\left(\triangle \mathrm{PA}=52^{\circ}\right.$ between $a=3^{\prime \prime}$ and $\left.12^{\prime \prime}\right)$ inside the large-scale bar $\left(e_{\max }=0.53\right.$ at $a=31^{\prime \prime}$; $\left.\mathrm{PA}=22^{\circ}\right)$. The galaxy is only moderately inclined $(I=$ $30^{\circ}$ ) and the twist almost disappears (reduces to $\triangle \mathrm{PA}=$ $\left.10^{\circ}\right)$ after deprojection.

NGC $4612\left(\mathrm{SB} 0+, 1^{\prime \prime} \sim 120 \mathrm{pc}, I=47.1^{\circ}\right)$ :

The isophotes of this lenticular galaxy severely twist $\left(\Delta \mathrm{PA}=48^{\circ}\right)$ between $a=2^{\prime \prime}$ and $a=15.7^{\prime \prime}$ where the ellipticity has a maximum $\left(e_{\max }=0.22\right)$. After deprojection $\left(\mathrm{PA}_{\text {disk }}=145^{\circ}\right)$, the ellipticity has a peak $\left(e_{\max }=0.4\right)$ along which the PA is roughly constant while a small twist $\left(\Delta \mathrm{PA}=14^{\circ}\right)$ still exists within the innermost $10^{\prime \prime}$. We interpret the profiles as resulting from the presence of a weak bar (we do not feel obvious to classify the galaxy as strongly barred as it is in RC3).

NGC $4665\left(\mathrm{SB} 0 / \mathrm{a}, 1^{\prime \prime} \sim 51 \mathrm{pc}, I=2.6^{\circ}\right)$ :

There is a small gradual twist along the large-scale bar ( $11^{\circ}$ between $a=3^{\prime \prime}$ and $42^{\prime \prime}$ where).

NGC 5643 (SABc/Seyfert 2, $\left.1^{\prime \prime} \sim 66 \mathrm{pc}, I=28.8^{\circ}\right)$ :

Despite the low disk inclination, there is a strong twist $\left(\triangle \mathrm{PA}=44^{\circ}\right.$ between $a=3^{\prime \prime}$ and $\left.30^{\prime \prime}\right)$ inside the largescale bar. This is interesting, because NGC 5643 belongs to late-type $(\mathrm{Sc})$ galaxies - which are expected not to show a twist (Elmegreen et al. 1996) - and, at the same time, has a Seyfert nucleus.

NGC 5701 (RSB0/a, $\left.1^{\prime \prime} \sim 100 \mathrm{pc}, I=15.2^{\circ}\right)$ :

There is a strong twist $\left(\triangle \mathrm{PA}=39^{\circ}\right.$ between $a=3^{\prime \prime}$ and $\left.38^{\prime \prime}\right)$ inside the large-scale bar $\left(e_{\max }=0.44\right.$ at $a=38^{\prime \prime}$, $\left.\mathrm{PA}=0^{\circ}\right)$, probably not explainable by projection effects since the galaxy inclination is only $15^{\circ}$.

\subsection{No nuclear twist or complex morphology}

NGC 1255 (SABbc, $\left.1^{\prime \prime} \sim 96 \mathrm{pc}, I=51.4^{\circ}\right)$ :

No evidence for twist is found; the first, rather flat peak in ellipticity $\left(e_{\max }=0.53\right.$ at $\left.a=4.4^{\prime \prime}\right)$ corresponds to the bar $\left(\mathrm{PA}=116^{\circ}\right)$, while the second, more pronounced (at $\left.a \sim 30^{\prime \prime}\right)$ is related to a double-armed spiral structure. The profiles are qualitatively similar after deprojection $\left(\mathrm{PA}_{\mathrm{disk}}=117^{\circ}\right)$.

NGC $1640\left(\mathrm{SBb}, 1^{\prime \prime} \sim 92 \mathrm{pc}, I=29.1^{\circ}\right)$ :

The PA is constant $\left(\sim 45^{\circ}\right)$ along the large-scale bar whose ellipticity maximum (0.62) is reached at $a=31^{\prime \prime}$.
NGC $1744\left(\mathrm{SBd}, 1^{\prime \prime} \sim 36 \mathrm{pc}, I=64.4^{\circ}\right)$ :

The galaxy is inclined at $I \sim 65^{\circ}$ and, moreover, our image is noisy: the ellipse fitting is not reliable. No clear evidence for twist is found.

NGC $1784\left(\mathrm{SBc}, 1^{\prime \prime} \sim 140 \mathrm{pc}, I=53.2^{\circ}\right)$ :

The ellipticity profile shows one maximum $\left(e_{\max }=0.68\right.$ at $\left.a=26^{\prime \prime}\right)$ near the end of the large-scale bar. The PA is well constant (between 91 and $95^{\circ}$ ) along the bar.

NGC 2911 (SA0/Seyfert 3, $1^{\prime \prime} \sim 200$ pc, $I=47.4^{\circ}$ ):

The PA, as well as the ellipticity, are rather flat inside $a=12^{\prime \prime}$. The small features further out are most likely due to badly masked stars.

NGC $3346\left(\mathrm{SBcd}, 1^{\prime \prime} \sim 82 \mathrm{pc}, I=20.1^{\circ}\right)$ :

No twist is found inside the primary bar $\left(e_{\max }=0.69\right.$ at $a=13^{\prime \prime}$ ).

NGC $4050\left(\mathrm{SBab}, 1^{\prime \prime} \sim 115 \mathrm{pc}, I=46.8^{\circ}\right)$ :

The PA is roughly constant $\left(77-87^{\circ}\right)$ along the large-scale bar.

NGC $4106\left(\mathrm{SB} 0+, 1^{\prime \prime} \sim 130 \mathrm{pc}, I=51.0^{\circ}\right)$ :

The PA is remarkably constant $\left(90-93^{\circ}\right)$ within $a=$ $11.5^{\prime \prime}$. The ellipticity has a maximum (0.37) inside that PA plateau (at $a=5.3^{\prime \prime}$ ). The PA grows later on while the ellipticity decreases.

NGC $4212\left(\mathrm{SAc}, I=51.5^{\circ}\right)$ :

The ellipticity peak near $a=29^{\prime \prime}$ is related to spiral arms, not to a bar structure. The inner isophotes, inside $10^{\prime \prime}$, show no clear twist: an oblate bulge is probably sufficient to explain the profiles.

NGC 4267 (SB0-, $\left.1^{\prime \prime} \sim 71 \mathrm{pc}, I=22.5^{\circ}\right)$ :

The PA is constant along the large-scale bar $\left(e_{\max }=0.22\right.$ at $a=18.4^{\prime \prime}, \mathrm{PA}=32^{\circ}$ ) of this low-inclination galaxy.

NGC $4424\left(\mathrm{SBa}, I=66.8^{\circ}\right)$ :

The galaxy is inclined at $I=67^{\circ}$. The twist inside the bar $\left(e_{\max }=0.78\right.$ reached at $\left.a=8^{\prime \prime} ; \mathrm{PA}=110^{\circ}\right)$ does not exceed $7^{\circ}$.

NGC 4501 (SAb/Seyfert 2, $\left.1^{\prime \prime} \sim 150 \mathrm{pc}, I=60.2^{\circ}\right)$ :

The bulge region is characterized by growing ellipticity. However, the constancy of the PA $\left(140-144^{\circ}\right.$ interior to $\left.a=18^{\prime \prime}\right)$ - as well as the fact that its value corresponds to that of the outer disk - is compatible with a spheroidal shape of the bulge: there is no evidence for a bar or other triaxiality. The change of eccentricity and PA behind $a=$ $18^{\prime \prime}$ reflects the presence of spiral arms.

NGC $4519\left(\mathrm{SBd}, 1^{\prime \prime} \sim 80 \mathrm{pc}, I=36.5^{\circ}\right)$ :

No isophotal twist is found inside the bar region of this late-type galaxy.

NGC 4689 (SAbc, $\left.1^{\prime \prime} \sim 110 \mathrm{pc}, I=33.0^{\circ}\right)$ :

Multiple features in the ellipticity and PA profiles result from flocculent spiral structure. No clear twist occurs in the nuclear region.

NGC $4731\left(\mathrm{SBcd}, 1^{\prime \prime} \sim 94 \mathrm{pc}, I=69.3^{\circ}\right)$ :

The galaxy is rather inclined and the ellipse fitting is unreliable. No clear twist is found.

NGC $4781\left(\mathrm{SBd}, 1^{\prime \prime} \sim 77 \mathrm{pc}, I=67.9^{\circ}\right)$ :

No clear twist is found inside the large-scale bar $\left(e_{\max }=\right.$ 
0.56 at $a=6.3^{\prime \prime}, \mathrm{PA}=90^{\circ}$ ). Spiral arms are responsible for features in the profile at larger distances.

NGC $4900\left(\mathrm{SBc}, 1^{\prime \prime} \sim 64 \mathrm{pc}, I=5.3^{\circ}\right)$ :

The galaxy is seen approximately face-on $\left(I=5.3^{\circ}\right)$ and no twist is found inside the large-scale bar $\left(e_{\max }=0.63\right.$ at $\left.a=4.9^{\prime \prime}, \mathrm{PA}=140^{\circ}\right)$. Further out, an irregular spiral structure causes changes in the ellipticity and PA profiles.

NGC $4902\left(\mathrm{SBb}, 1^{\prime \prime} \sim 170 \mathrm{pc}, I=25.1^{\circ}\right)$ :

The PA inside the large-scale bar $\left(e_{\max }=0.60\right.$ at $\left.a=25^{\prime \prime}\right)$ is approximately constant (within $10^{\circ}$ ).

NGC $5236\left(\mathbf{M ~ 8 3 )}\left(\mathrm{SABc}, I=21.0^{\circ}\right)\right.$ :

The nuclear structure of this nearby "hot-spot" starburst galaxy (e.g. Telesco et al. 1993) is complex also in our $J H K$ images and the ellipse fitting in the central region not too meaningful (it even fails in $J$ ). The structure, patchy but dissimilar from optical and mid-IR will be analyzed elsewhere.

NGC 6753 (RSAb, $\left.1^{\prime \prime} \sim 190 \mathrm{pc}, I=29.3\right)$ :

The ellipticity and PA profiles of this galaxy show several small peaks related probably to the presence of star forming regions and flocculent spiral structure of the outer disk, as W95 have already noted. No clear triaxial feature can be found.

IC $1953\left(\mathrm{SBd}, 1^{\prime \prime} \sim 110 \mathrm{pc}, I=44.0^{\circ}\right)$ :

The PA is constant inside the large-scale bar $\left(e_{\max }=0.70\right.$ at $a=23^{\prime \prime}, \mathrm{PA}=156^{\circ}$ ).

Table 3. Projected parameters of single bars ( $H$ band)

$\begin{array}{llrrrr}\text { Galaxy } & e_{\max } & \begin{array}{r}l_{\max } \\ \left(^{\prime \prime}\right)\end{array} & \begin{array}{r}\text { PA } \\ \left({ }^{\circ}\right)\end{array} & \begin{array}{r}\text { twist } \\ \left({ }^{\circ}\right)\end{array} & \begin{array}{r}\text { tw. scale } \\ \left({ }^{\prime \prime}\right)\end{array} \\ \text { N 1187 } & 0.59 & 32 & 132 & 14 & 3-7 \\ \text { N 1255 } & 0.53 & 4.4 & 116 & \text { no } & - \\ \text { N 1302 } & 0.35 & 29 & 170 & 20 & 7-29 \\ \text { N 1640 } & 0.62 & 31 & 45 & \text { no } & - \\ \text { N 1784 } & 0.68 & 26 & 92 & \text { no } & - \\ \text { N 1792 } & 0.45 & 5 & & \text { no } & - \\ \text { N 1832 } & 0.58 & 17 & 166 & 14 & 3-11 \\ \text { N 2525 } & 0.69 & 22 & 74 & 13 & 3-22 \\ \text { N 3166 } & 0.16 & 18 & 170 & 47 & 13-17 \\ \text { N 3346 } & 0.69 & 14 & 97 & \text { no } & - \\ \text { N 3637 } & 0.38 & 15 & 37 & 71 & 3-15 \\ \text { N 3673 } & 0.73 & 42 & 87 & 17 & 3-42 \\ \text { N 3887 } & 0.68 & 33 & 0 & 22 & 3-25 \\ \text { N 4050 } & 0.70 ? & 69 ? & 79 & \text { no } & - \\ \text { N 4106 } & 0.37 & 5.3 & 93 & \text { no } & - \\ \text { N 4267 } & 0.22 & 18 & 32 & \text { no } & - \\ \text { N 4424 } & 0.78 & 8 & 110 & \text { no } & - \\ \text { N 4454 } & 0.53 & 31 & 22 & 52 & 3-12 \\ \text { N 4519 } & 0.58 & 3 & 78 & \text { no } & - \\ \text { N 4612 } & 0.21 & 16 & 98 & 46 & 3-16 \\ \text { N 4665 } & 0.53 & 42 & 3 & 11 & 3-42 \\ \text { N 4781 } & 0.56 & 6.3 & 90 & \text { no } & - \\ \text { N 4900 } & 0.63 & 4.9 & 140 & \text { no } & - \\ \text { N 4902 } & 0.60 & 25 & 67 & \text { no } & - \\ \text { N 5643 } & 0.68 ? & 49 ? & 87 & 44 & 3-30 \\ \text { N 5701 } & 0.44 & 38 & 0 & 39 & 3-39 \\ \text { IC 1953 } & 0.70 & 23 & 156 & \text { no } & -\end{array}$

\subsection{Highly inclined galaxies}

Galaxies listed below have the inclination higher than $75^{\circ}$. We publish their contour plots and ellipse fitting profiles but do not attempt to interpret them:

NGC $1518\left(\right.$ SBdm, $1^{\prime \prime} \sim 49$ pc, $\left.I=79.8^{\circ}\right)$ : NGC $2811\left(\mathrm{SBa}, 1^{\prime \prime} \sim 152 \mathrm{pc}, I=85.9^{\circ}\right)$, NGC 3384 (SB0-/AGN?, $\left.1^{\prime \prime} \sim 48 \mathrm{pc}, I=90^{\circ}\right)$, NGC 3593 (SA0/a, $\left.1^{\prime \prime} \sim 42 \mathrm{pc}, I=76.4^{\circ}\right)$, NGC $3885\left(\mathrm{SA} 0 / \mathrm{a}, 1^{\prime \prime} \sim 105 \mathrm{pc}\right.$, $\left.I=90^{\circ}\right)$, NGC $4178\left(\mathrm{SBdm}, I=90.0^{\circ}\right)$, NGC 4192 $\left(\mathrm{SABab} /\right.$ Seyfert $\left.3, I=90.0^{\circ}\right)$, NGC $4216(\mathrm{SABb}, I=$ $90.0^{\circ}$ ), NGC $4442\left(\mathrm{SB} 0,1^{\prime \prime} \sim 40 \mathrm{pc}, I=90.0^{\circ}\right.$ ), NGC $4461\left(\mathrm{SB} 0+, 1^{\prime \prime} \sim 120 \mathrm{pc}, I=85.7^{\circ}\right)$, NGC 4503 $\left(\mathrm{SB} 0-, 1^{\prime \prime} \sim 90 \mathrm{pc}, I=90.0^{\circ}\right)$, NGC 4546 (SB0-, $\left.1^{\prime \prime} \sim 67 \mathrm{pc}, I=90.0^{\circ}\right)$, NGC $4684\left(\mathrm{SB} 0+, 1^{\prime \prime} \sim 100 \mathrm{pc}\right.$, $\left.I=90.0^{\circ}\right)$, NGC $4694\left(\mathrm{SB} 0 / \mathrm{HII}, 1^{\prime \prime} \sim 79 \mathrm{pc}, I=79.7^{\circ}\right)$, NGC $4856\left(\mathrm{SB} 0 / \mathrm{a}, 1^{\prime \prime} \sim 82 \mathrm{pc}, I=90.0^{\circ}\right)$, NGC 6810 $\left(\mathrm{SAab}, 1^{\prime \prime} \sim 110 \mathrm{pc}, I=86.2^{\circ}\right)$.

\section{Conclusions}

A sub-sample of 56 galaxies whose nuclear structures were interpreted in terms of the ellipse fitting on near-IR images was constructed (16 other galaxies of the survey are seen nearly edge-on and were not analyzed in detail). Our principal results are the following.

1. We classify 17 galaxies of the subsample as having two triaxial structures, either double bars or a bar with an embedded triaxial bulge (some cases are speculative because of low-central resolution or high galactic inclination). Two of them (NGC 1433 and 6782) were known to possess a double-barred morphology from previous surveys in other colors, 15 detections are new (NGC 613, 1079, 1353, 1365, 1398, 1808, 1512, 2217, 2935, 3368, 3393, 4984, 5101, 5566 and ESO 437-67).

2. We find 16 other nuclear isophotal twists not associated with a clear ellipticity maximum (14 of them are new: NGC 1187, 1832, 2442, 2525, 2997, 3166, 3637, 3673, 3887, 4454, 4612, 4665, 5643, 5701; twists in NGC 1302 and 1512 were known from other bands).

3. We detect central triaxial features (nuclear bars or triaxial bulges) in three galaxies classified as SA in RC3: NGC 1792, 4438 and 5427. On the other hand, we find no evidence for triaxiality in the following SA's: NGC 2911, 4212, 4501, 4689 and 6753.

4. Four of our 17 double-barred galaxies host a Seyfert nucleus: NGC 613, 1365, 1808, 3393 (Seyfert activity of NGC 1808 is controversial).

5. Among 9 Seyferts from the sub-sample, there are 4 with a double bar (see item 4), 2 with a nuclear bar (NGC 4438 and 5427), 1 with a strong twist along its large-scale bar (NGC 5643) and 2 show no clear twist (NGC 2911 and 4501). 
6. Nine of our double-barred galaxies are known to have a nuclear ring (NGC 1433, 1512, 1808, 2935, 4984, 6782 and ESO 437-67) or a nuclear spiral (NGC 613 and 1365).

7. Among 32 galaxies with double barred structure or twist, 29 are of early Hubble types (S0-Sc); only 3 are of late type Sc: NGC 1187, 2525, 2997, 5643. Twists in the first 3 Sc's are small $\left(10-20^{\circ}\right)$ and can be caused by projection effects; the twist in NGC 5643 - which is a Seyfert emitter - is strong and probably intrinsic. No Sc galaxy with a twist has been known so far.

Comparison of the present survey with photometry in other colors and with kinematical data, as well as the interpretation, will be done in a separate paper.

Acknowledgements. The authors acknowledge the participation of M. Shaw on the selection of the observed sample of galaxies. B.J. wishes to express many thanks to P. Lena, J.-P. Zahn and J. Palous for their efforts in co-organizing the $\mathrm{PhD}$ "cotutelle" between University Paris VII and Charles University in Prague, and, especially, to F. Combes for having accepted to co-direct his PhD thesis. He also acknowledges useful discussions on data reduction techniques with J.M. Deltorn as well as the help of H. Flores with software problems. The stay of B.J. at the Paris Observatory was supported by a scholarship of the French Government.

\section{References}

Bender R., Möllenhoff, 1987, A\&A 177, 71

Bertola F., Galletta G., 1979, A\&A 77, 363

Buta R., 1986, ApJS 61, 631

Buta R., 1990, ApJ 351, 62

Buta R., Crocker D.A., 1993, AJ 105/4, 1344

Combes F., 1994, in: Mass-transfer induced Activity in Galaxies, Shlosman I. (ed.). Cambridge University Press, p. 170

Combes F., Debbasch F., Friedli D., Pfenninger D., 1990, A\&A 233, 82

Combes F., Dupraz C., Casoli F., Pagani L., 1988, A\&A 203, L9

Davies C.L., Hunter J.H. Jr., 1996, in: Waves in Astrophysics, Hunter J.H. Jr., Wilson R.E. (eds.). New York Academy of Sciences

de Jong R.S., van der Kruit P.C., 1994, A\&AS 106, 451

de Vaucouleurs G.A., 1974, in: Formation of Galaxies, IAU Symp. No. 58, Shakeshaft J.R. (ed.). Reidel, Dordrecht, p. 335

de Vaucouleurs G.A., de Vaucouleurs H.G., Corwin R.J., Buta G., Paturel G., Fouqué P., 1993, Third Reference Catalogue of Bright Galaxies. Springer, New York
Devereux N., 1987, ApJ 323, 91

Devereux N., 1989, ApJ 346, 126

Elmegreen D.M., Elmegreen B.G, Chromey F.R., Hasselbacher D.A., Bissell B.A., 1996, AJ 111/5, 1880

Forbes D.A., Ward M.J., DePoy D.L., Boisson C., Smith M.S., 1992, MNRAS 254, 509

Friedli D., 1996, A\&A 312, 761

Friedli D., Wozniak H., Rieke M., Martinet L., Bratschi P., 1996, A\&AS 118, 461

Friedli D., Benz W., 1993, A\&A 268, 65

Friedli D., Martinet L., 1993, A\&A 227, 27

Griersmith D., Hyland A.R., Jones T.J., 1982, AJ 87/8, 1106

Héraudeau Ph., Simien F., Mamon G.A., 1996, A\&AS 117, 417

Hummel E., van der Hulst J.M., Keel W.C., 1987, A\&A 172, 32

Jarvis B., Dubath P., Martinet L., Bacon R., 1988, A\&AS 74, 513

Kenney J.D.P., Rubin, V.C., Planesas P., Young J.S., 1995, ApJ 438

Kormendy J., 1979, ApJ 227, 714

Kormendy J., 1982, ApJ 257, 75

Kotilainen J.K., Forbes D.A., Moorwood A., van der Werf P.P., Ward J.M., 1996, A\&A 313, 771

Liller M.H., 1960, ApJ 132, 306

Madejsky R., Möllenhoff C., 1990, A\&A 234, 119

Martin P., 1995, AJ 109/6, 2428

McLeod K.K., Rieke G.H., 1995, ApJ 441, 96

Nieto J.-L., Bender R., Poulain P., Surma P., 1992, A\&A 257, 97

Pompea S., Rieke G.H., 1990, ApJ 356, 416

Phillips A.C., 1993, AJ 105, 486

Rauscher B., 1995, AJ 109/4, 1609

Saikia D.J., Unger S.W, Pedler A., Yates G.J., Axon D.J., Wolstencroft R.D., Taylor K., Gyldenkerne K., 1990, MNRAS 245, 397

Sandqvist A., Jörsäter S., Lindblad P.O., 1995, A\&A 295, 585

Shaw M.A., Axon D., Probst R., Gatley I., 1995, MNRAS 274, 369

Shaw M.A., Combes F., Axon D.J., G.S. Wright, 1993, A\&A 273,31

Shlosman I., Frank J., Begelman M., 1989, Nat 338, 45

Tacconi-Garman L., Sternberg A., Eckart A., 1996, AJ 112, 918

Telesco C.M., Dressel L.L., Wolstencroft R.D., 1993, ApJ 414, 120

Teuben P.J., Sanders R.H., Atherton P.D., van Albada G.D., 1986, MNRAS 221, 1

Véron-Cetty M.-P., Véron P., 1985, A\&A 145, 425

Véron-Cetty M.-P., Véron P., 1996, Quasars and Active Galactic Nuclei, 7th ed., ESO Scientific Report, Garching

Wozniak H., Friedli D., Martinet L., Martin P., Bratschi P., 1995, A\&AS 111, 115 


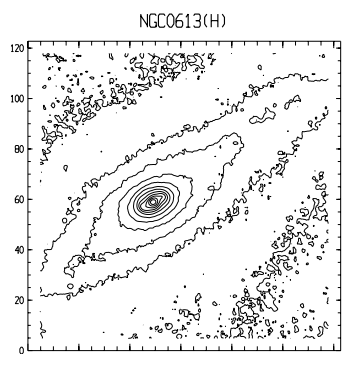

NGC1079(H)

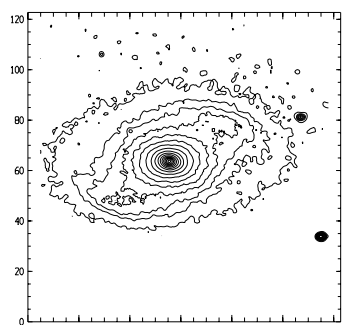

NGC1187(H)

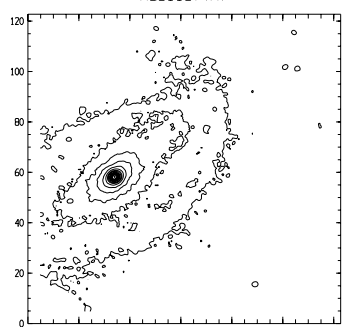

$\mathrm{NGC1255(H)}$

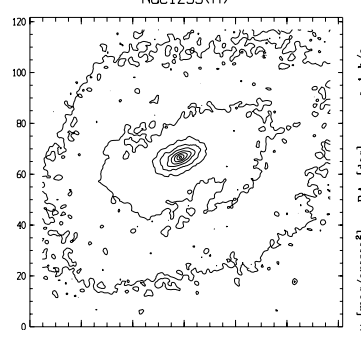

NGC1302(H)

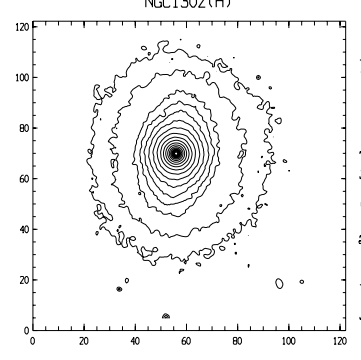

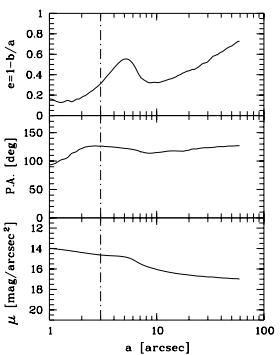

a [arcsec
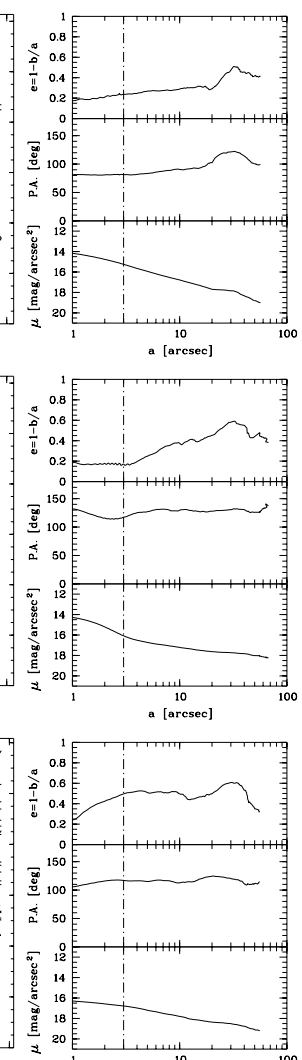

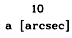

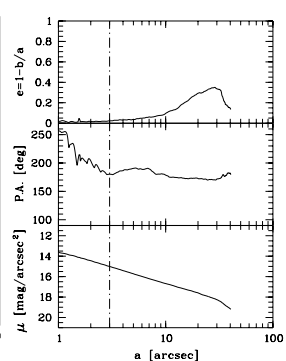

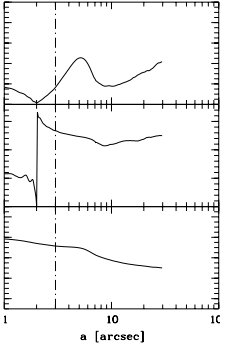
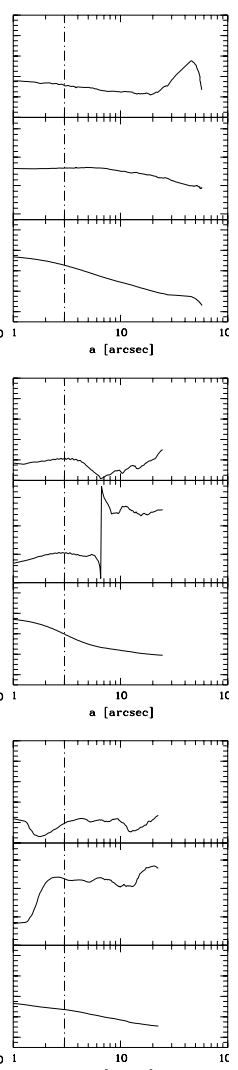

a [arcsec]

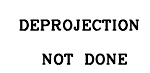

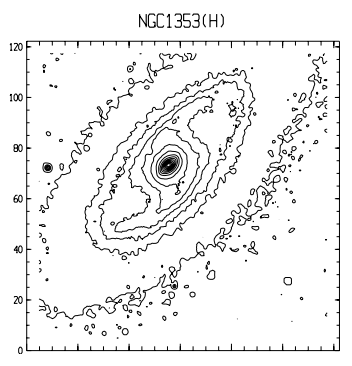

$\mathrm{NGC1365(H)}$

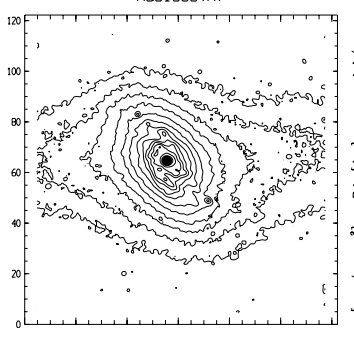

NGC1433(H)

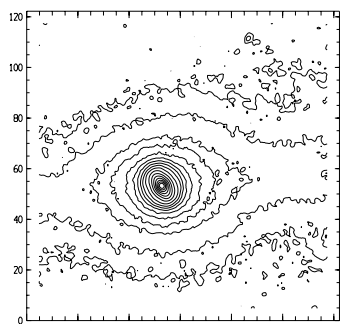

$\operatorname{NGC1433(J)}$

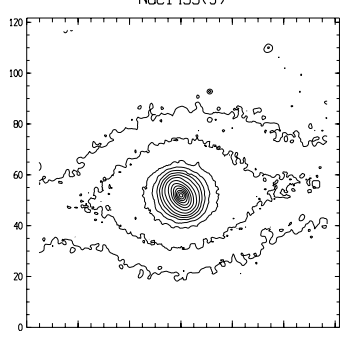

NGC1433(K)

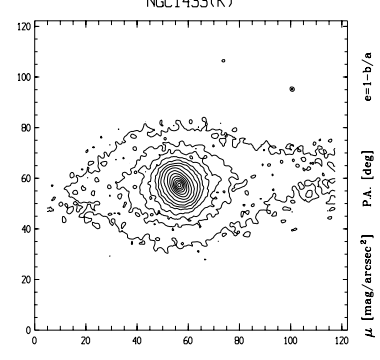

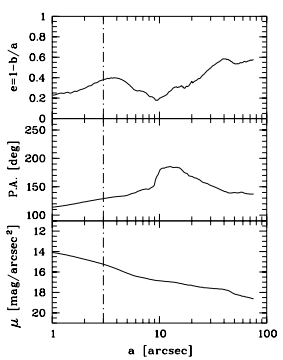
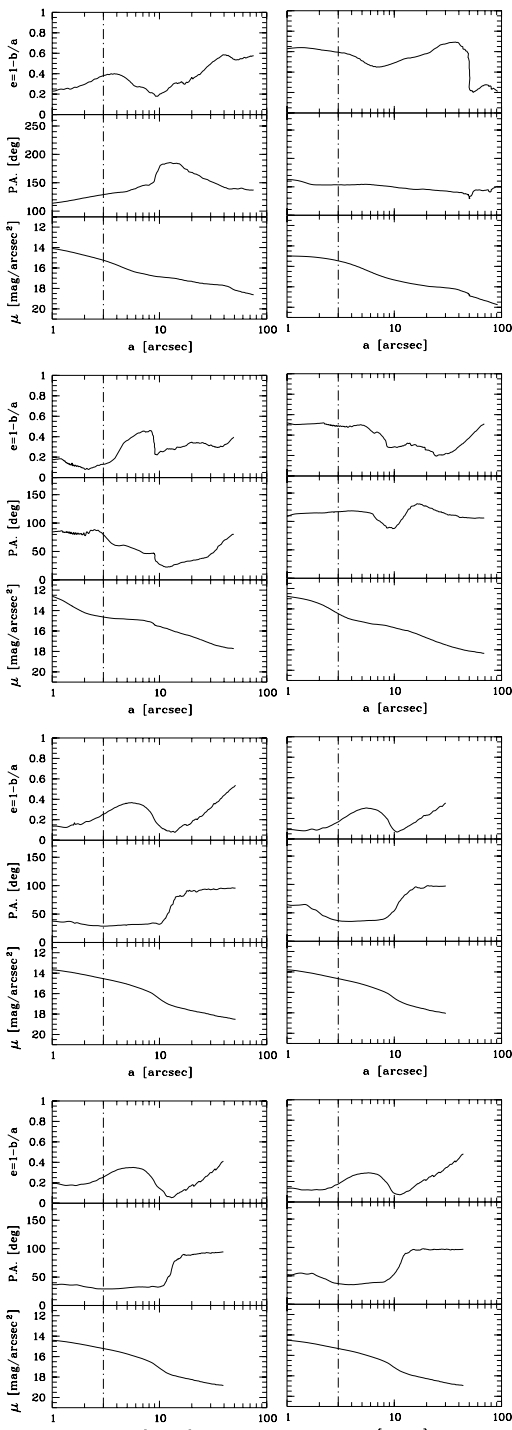

a [arcsec]
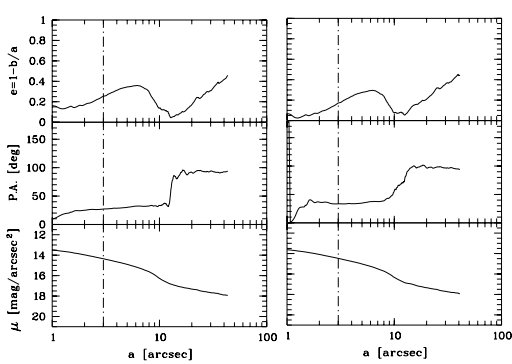

Fig. 4. Contour plots and ellipse fitting. The left box shows logarithmically scaled intensity map on the area of $120 \times 120$ arcseconds; the North is at the top, the East at the left. The ellipse fitting output - radial profiles of ellipticity, position angle and surface brightness - is displayed in the central plot; the abscissa - semi-major axis of fitted ellipses - is scaled logarithmically. The right plot shows the same quantities after deprojection. The vertical dash-dot lines in radial profile plots separate the innermost region of $a<3^{\prime \prime}$ where the ellipse fitting is judged to be unreliable 

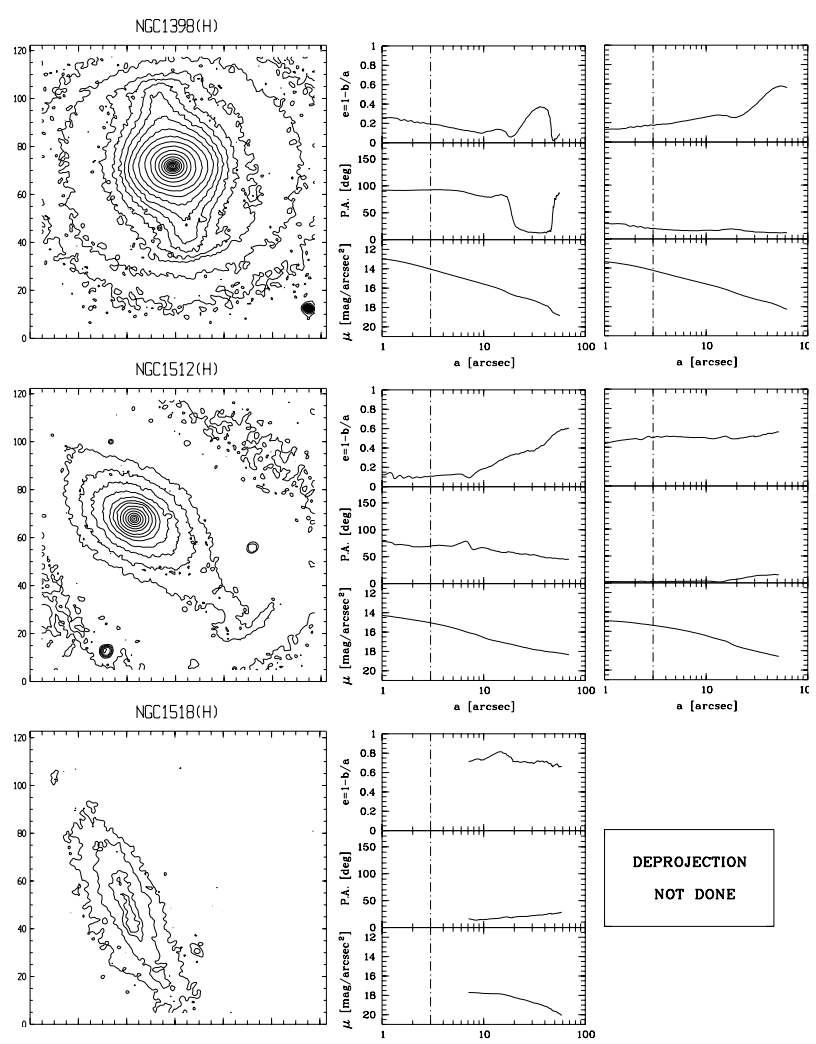

NGC1640(H)
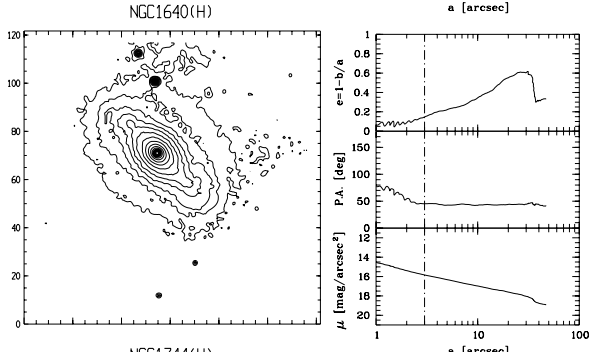

NGC1744(H)

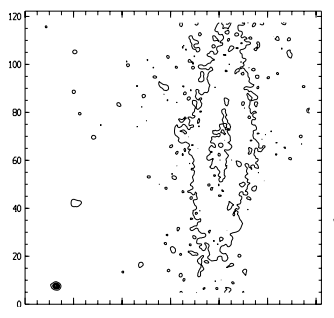

$\mathrm{NGC1784( \textrm {H } )}$
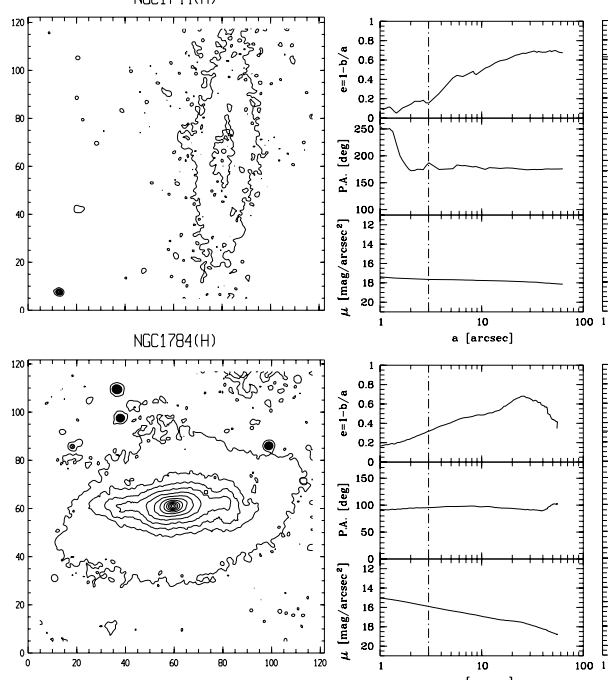

a [arcsec]
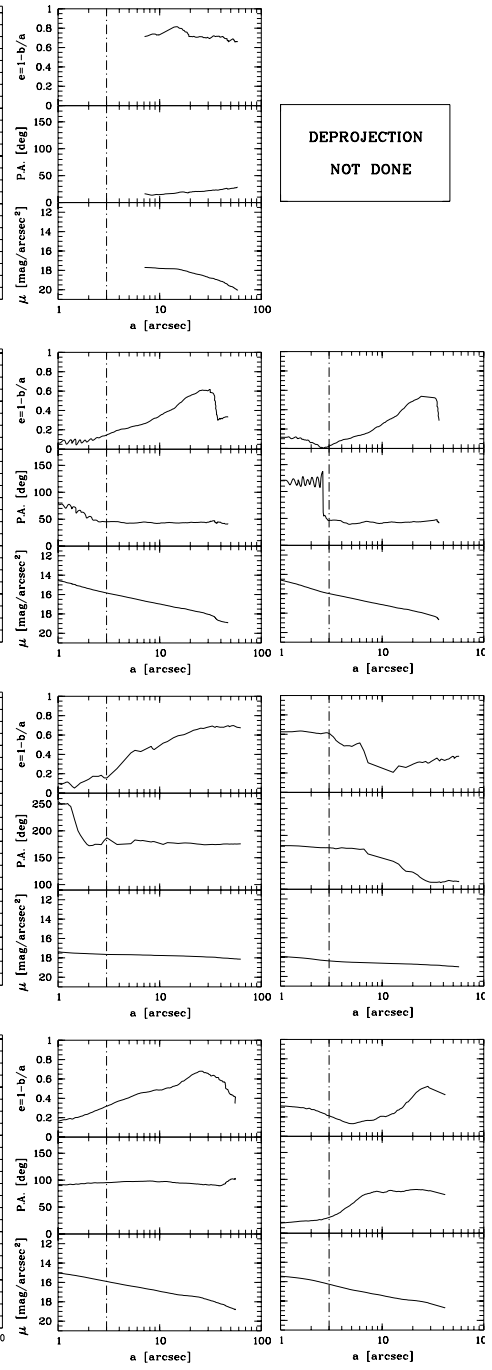
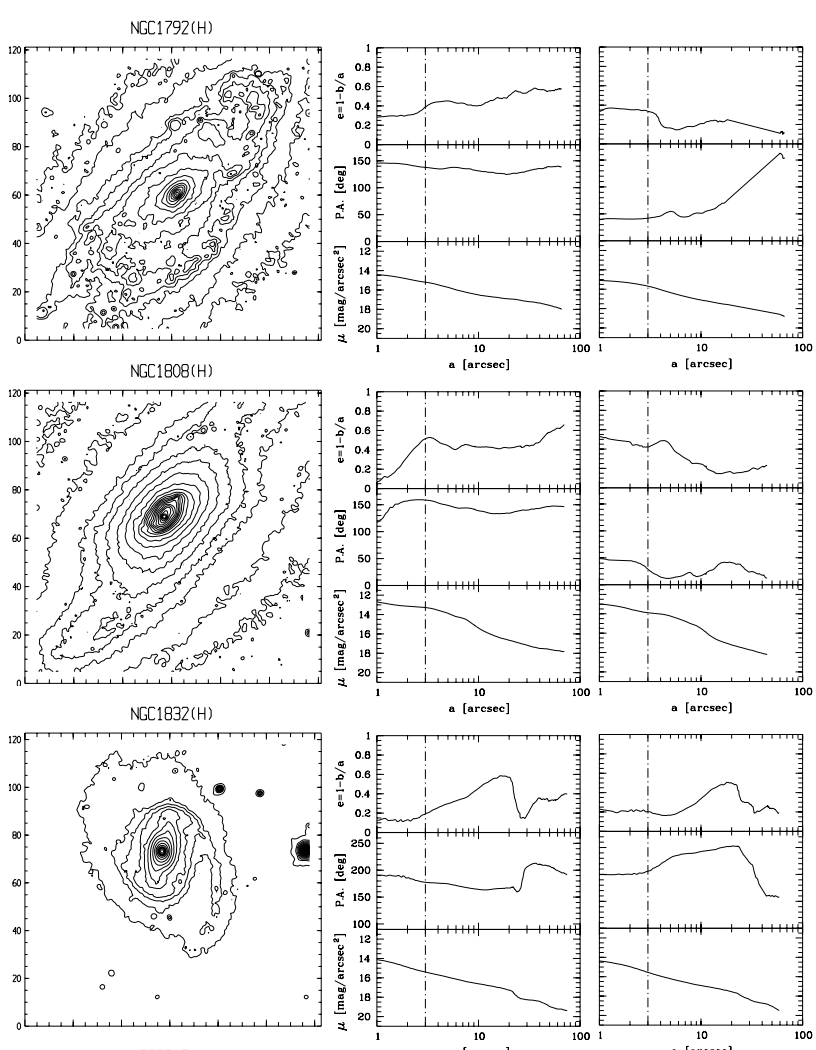

NGC2217(H)
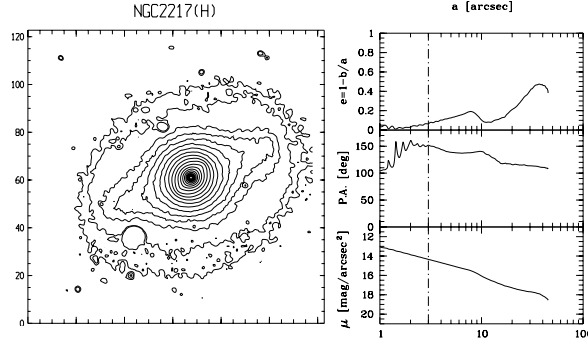

DEPROJECTION

NOT DONE
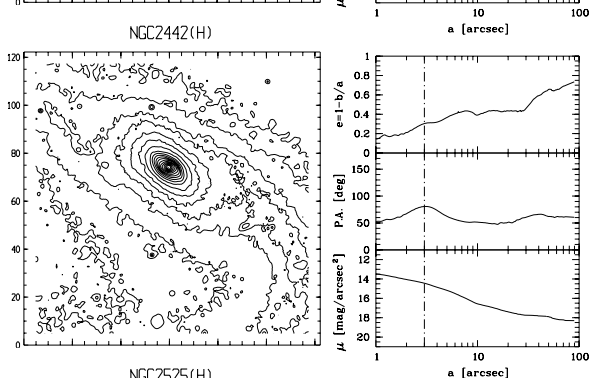

DEPROJECTION

NOT DONE

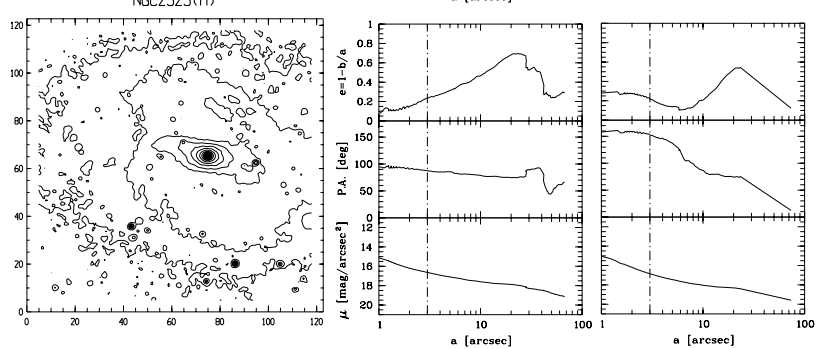

Fig. 4. continued 


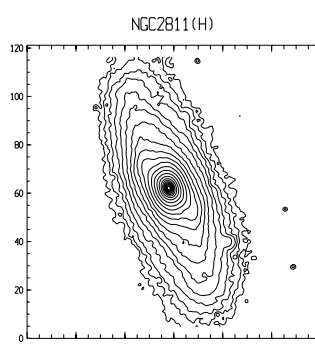

NGC2911(H)

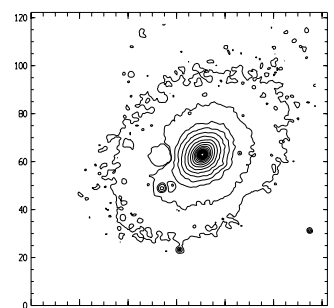

$\mathrm{NGC2935(H)}$

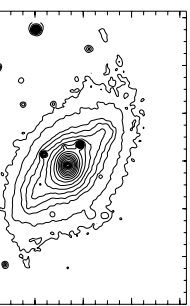

NGC2997(H)

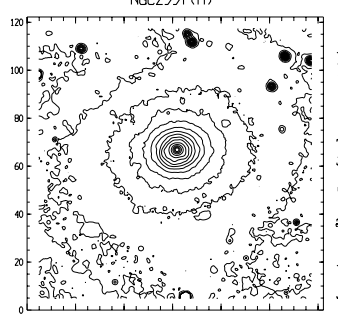

NGC3166(H)

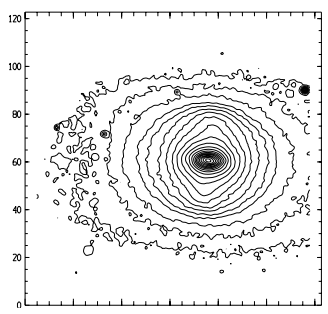

$\operatorname{NGC3368(H)}$

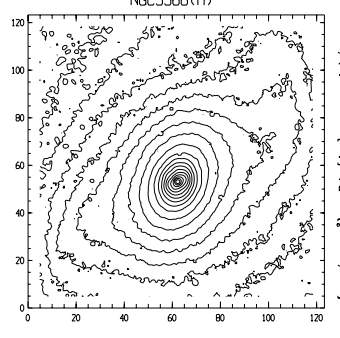

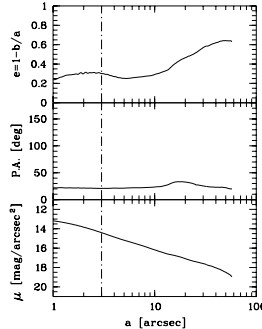

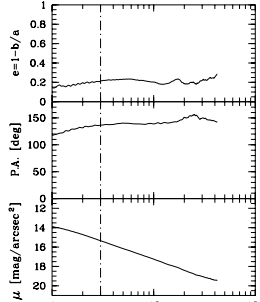

$\underset{\text { a [arcsec] }}{10}$

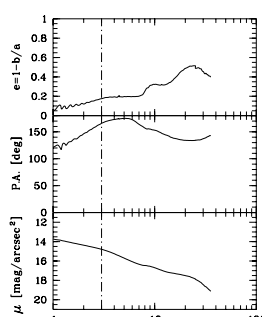

a [arcsec]

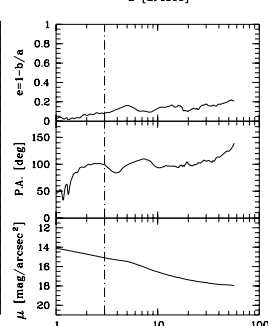

10
a [arcsec]

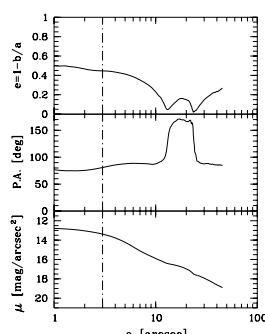

10
a [arcsec]

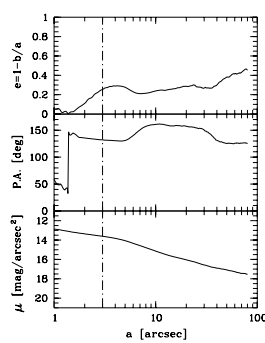

DEPROJECTION NOT DONE

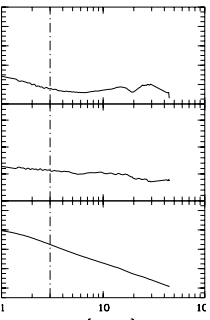

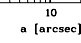

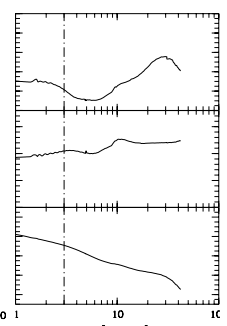

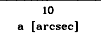
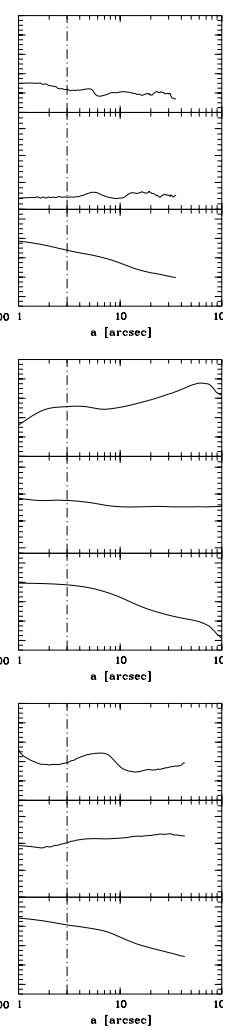

NGC3346(H)
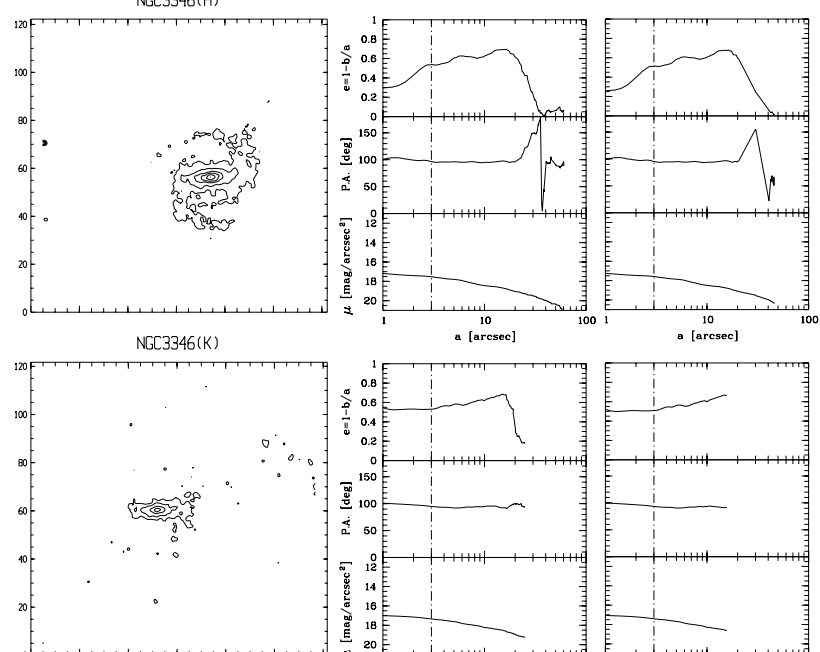

NGC3384(H)

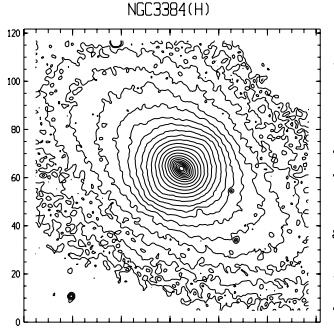

NGC3384(J)

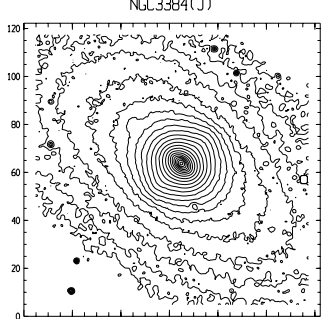

NGC3593(J)

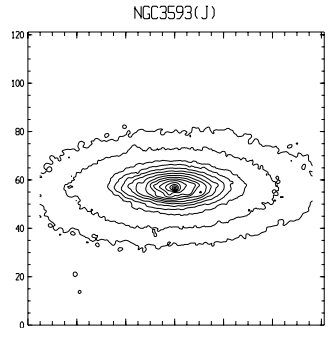

NGC3593(H)

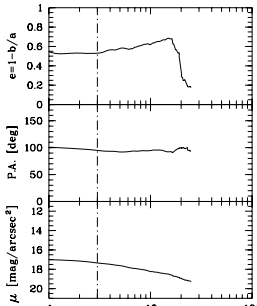

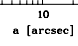
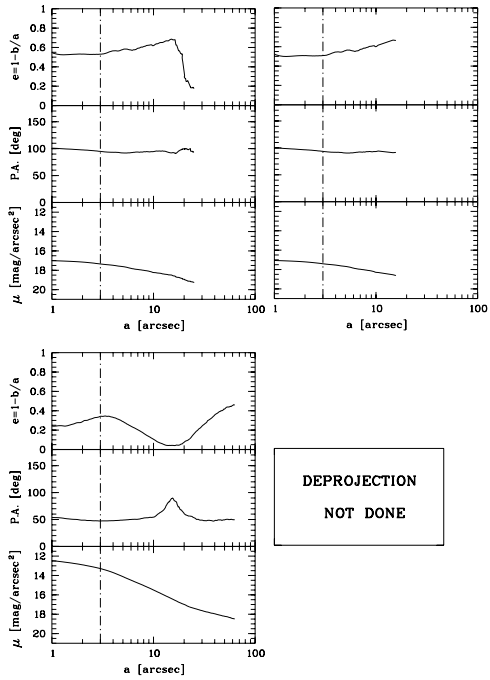

DEPROJECTION

NOT DONE

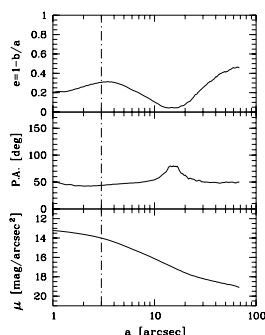

DEPROJECTION

NOT DONE

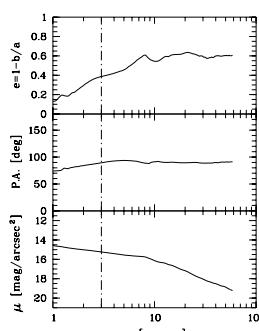

10
a [arcsec]
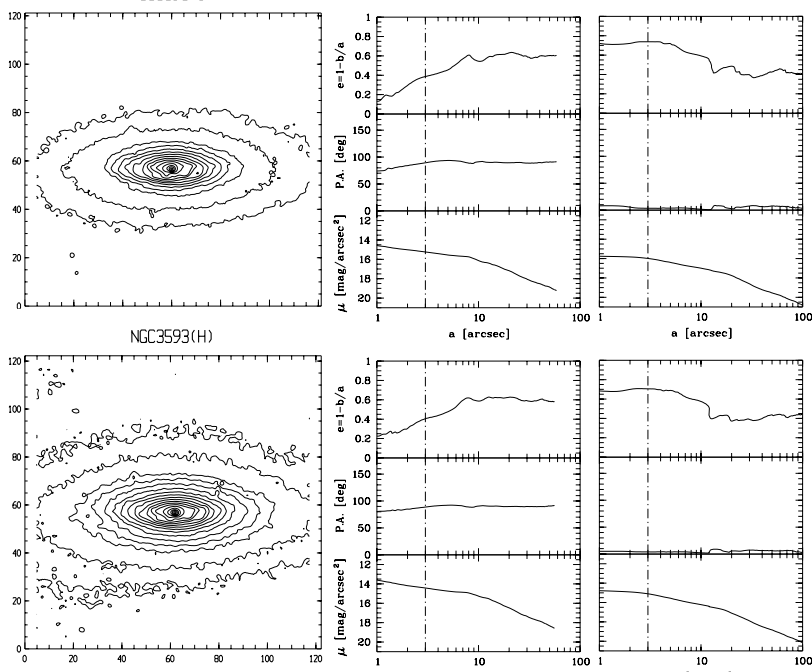
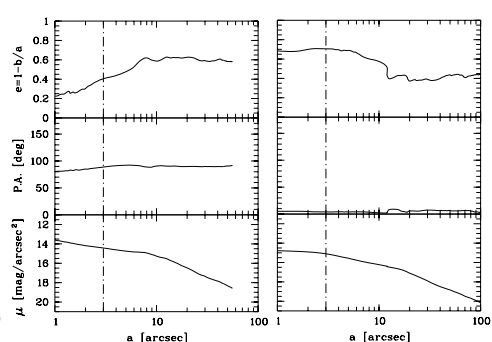

Fig. 4. continued 

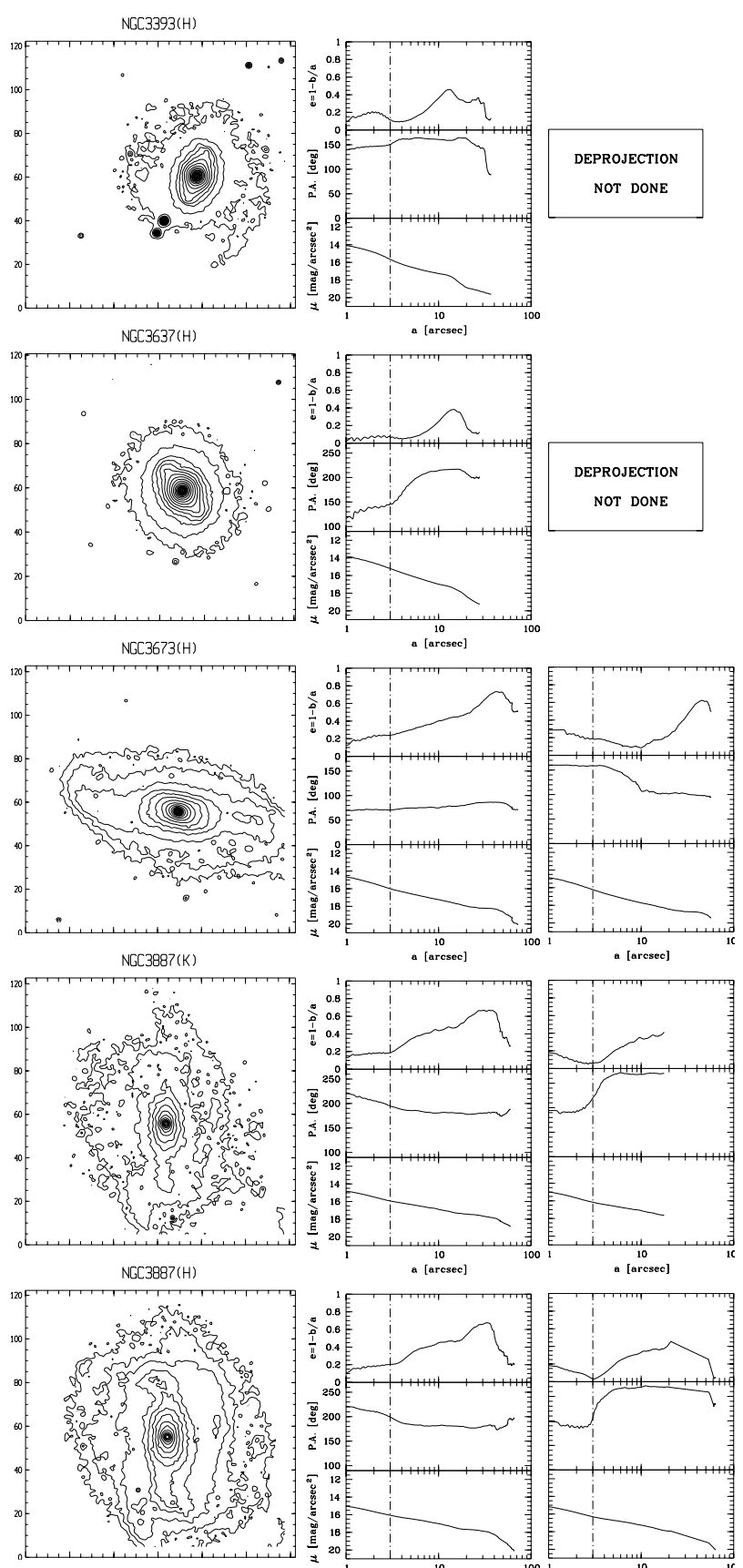

$\mathrm{NGC3887(J)}$
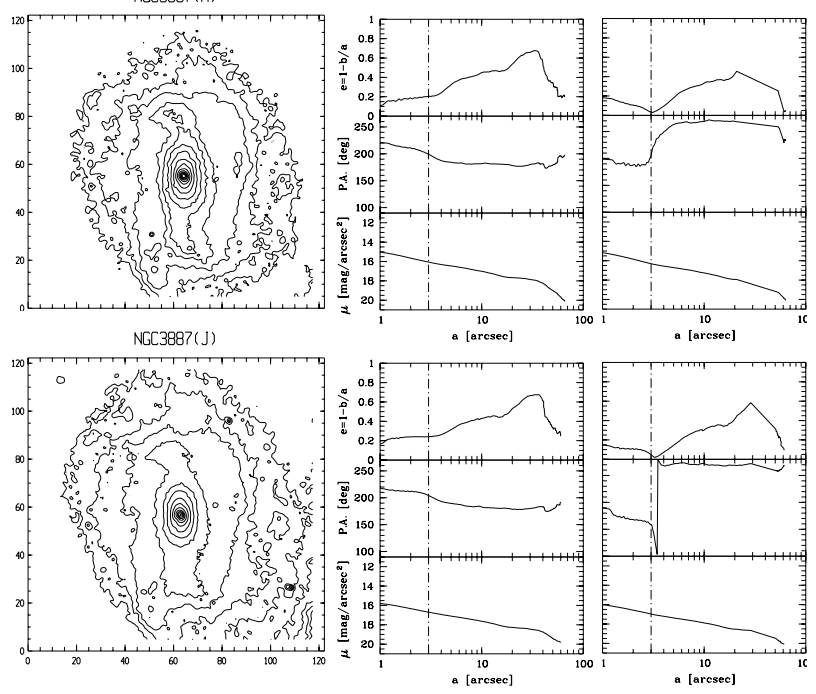
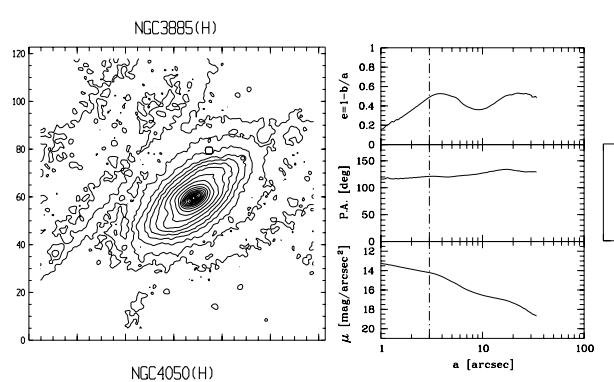

DEPROJECTION NOT DONE

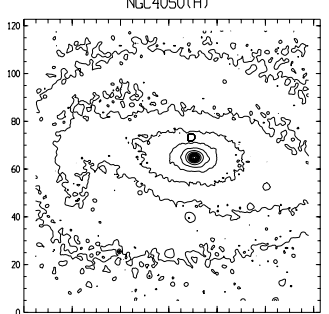

$\mathrm{NGC4106(H)}$
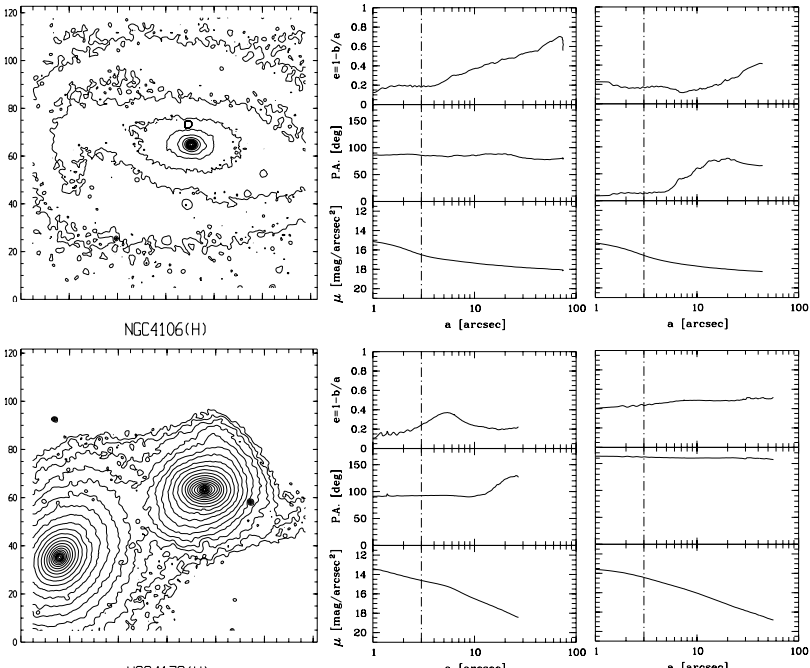

NGC4178(H)

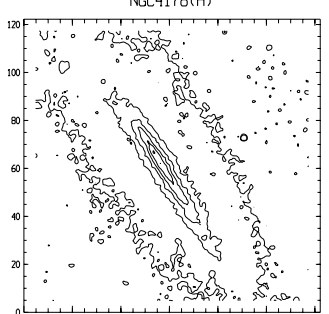

NGC4192(H)
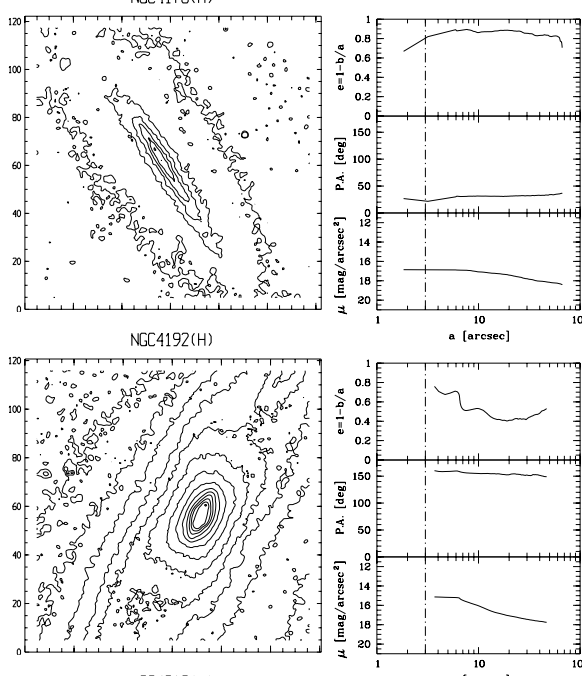

DEPROJECTION

NOT DONE

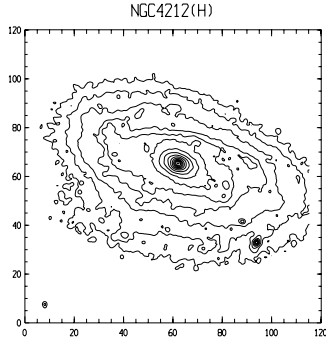

DEPROJECTION

NOT DONE

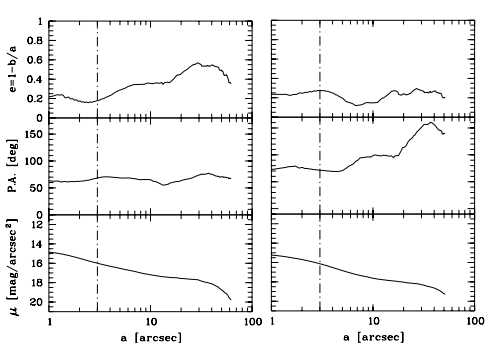

Fig. 4. continued 

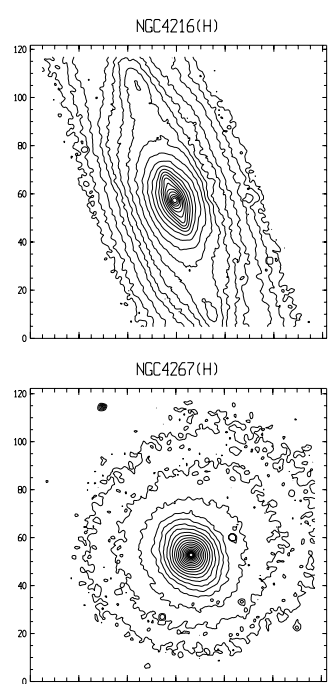

NGC4424(H)

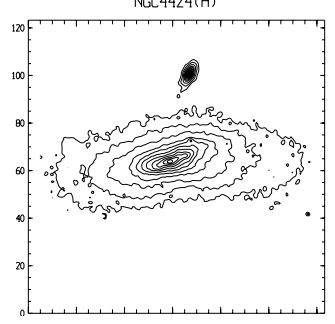

NGC4438(H)

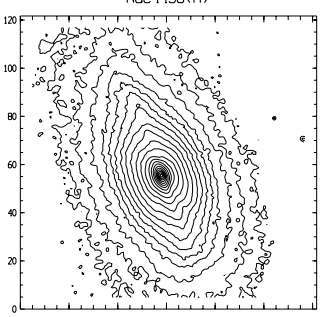

NGC4442(H)

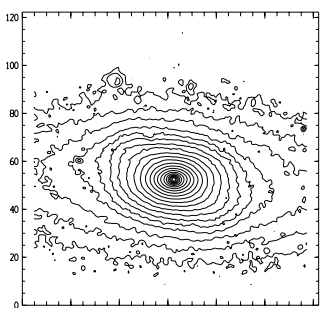

NGC 4454(H)

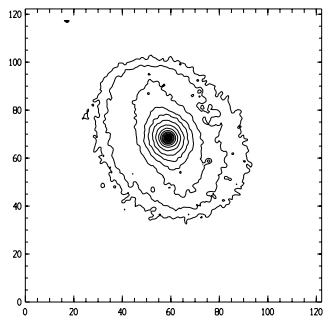

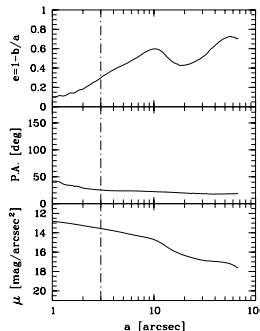
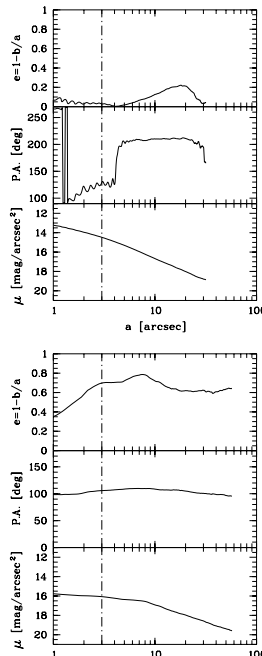

a [arcsec]
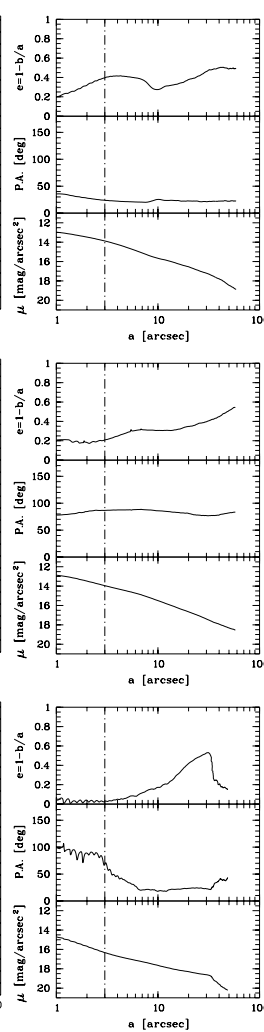

DEPROJECTION NOT DONE

DEPROJECTION NOT DONE

DEPROJECTION NOT DONE

DEPROJECTION NOT DONE
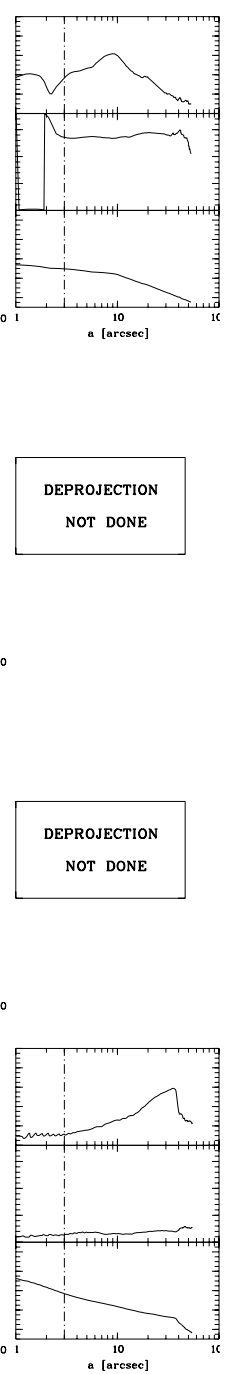
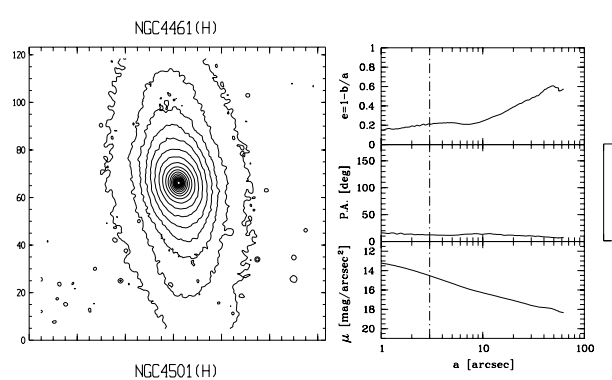

DEPROJECTION NOT DONE
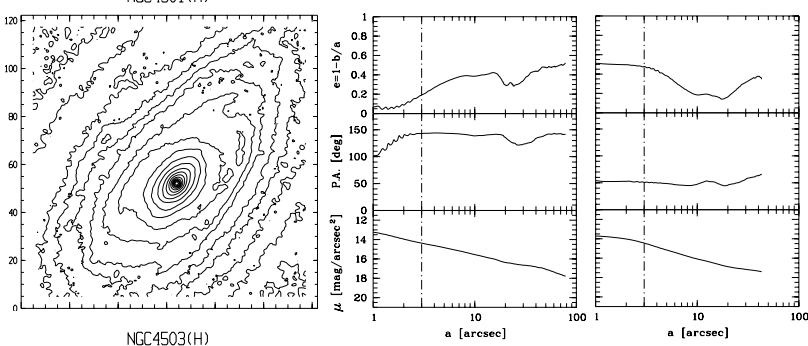

NGC4503(H)

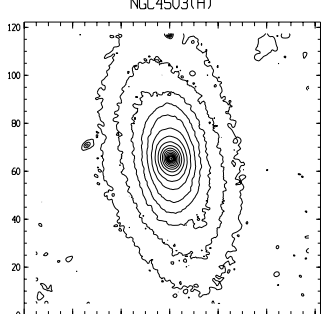

NGC4519(H)

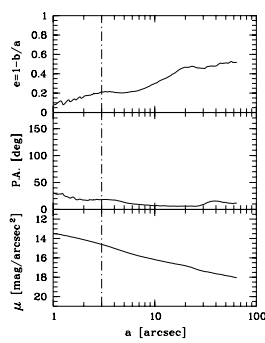

DEPROJECTION NOT DONE
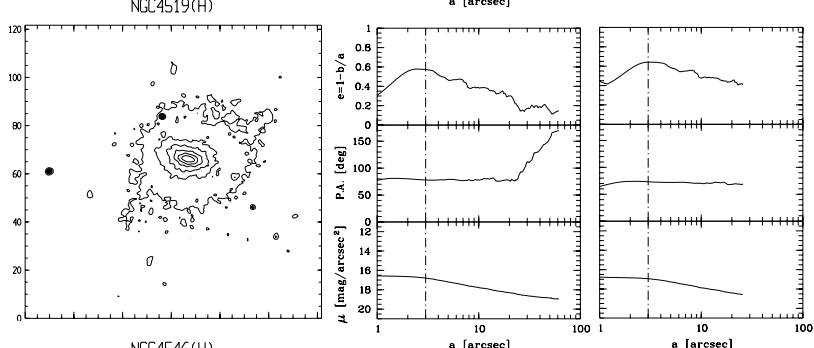

NGC 4546(H)
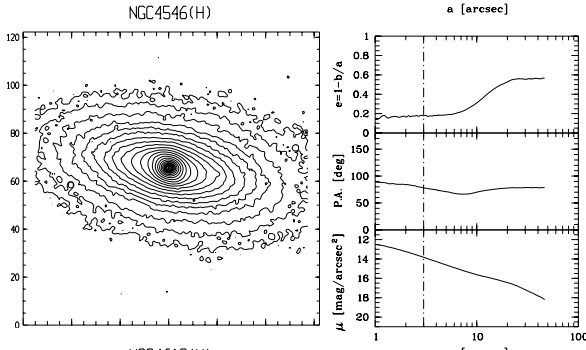

DEPROJECTION NOT DONE
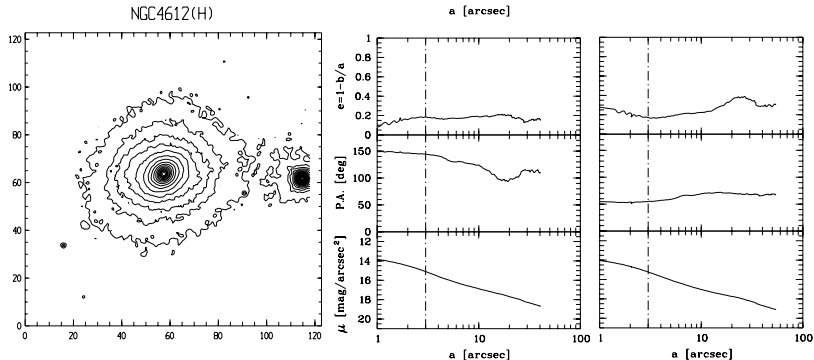

Fig. 4. continued 

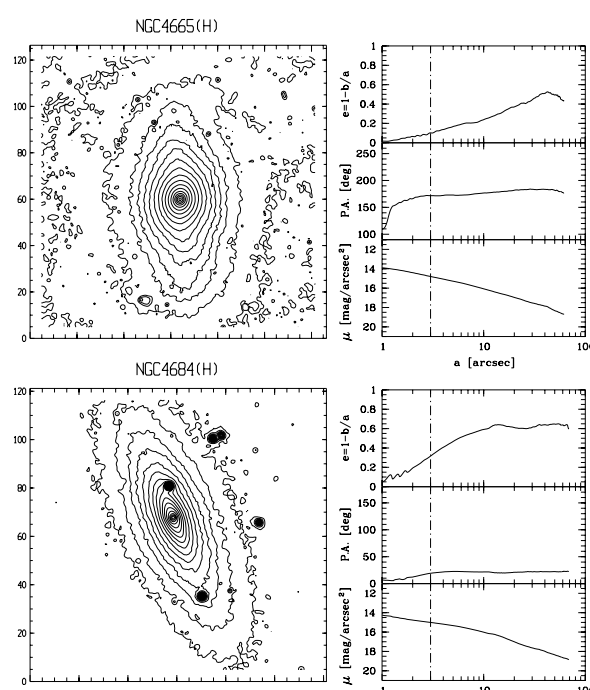

NCC 4689(H)

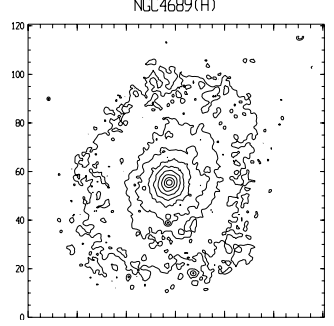

NGC 4694(H)

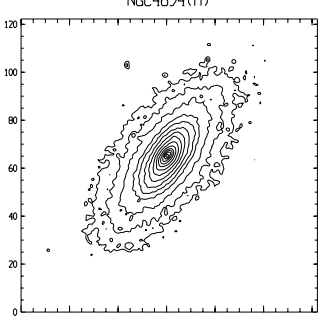

NGC4731 (H)

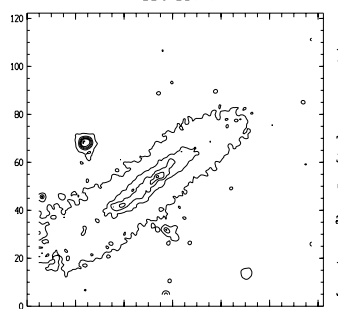

NGC4781 (H)

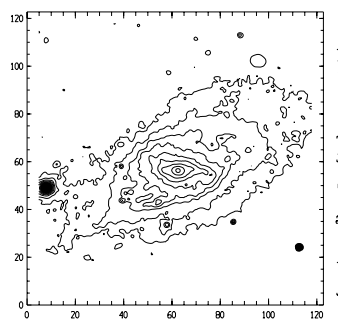

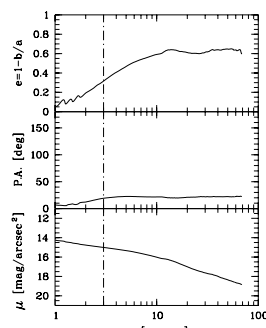

DEPROJECTION NOT DONE

DEPROJECTION NOT DONE
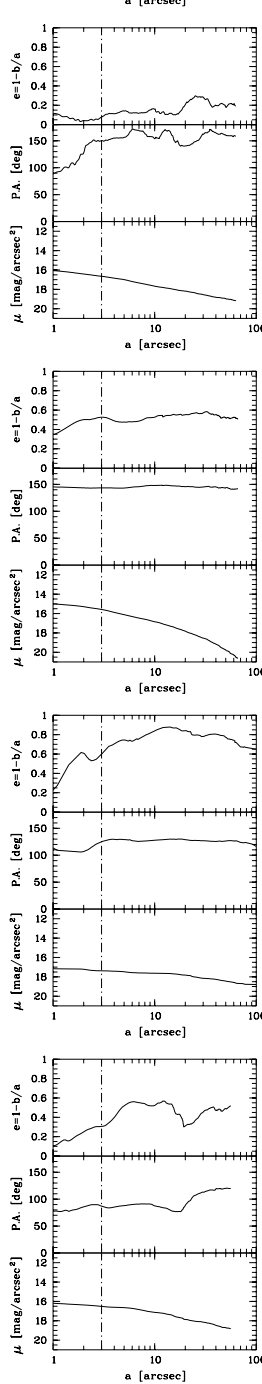

DEPROJECTION NOT DONE
DEPROJECTION NOT DONE
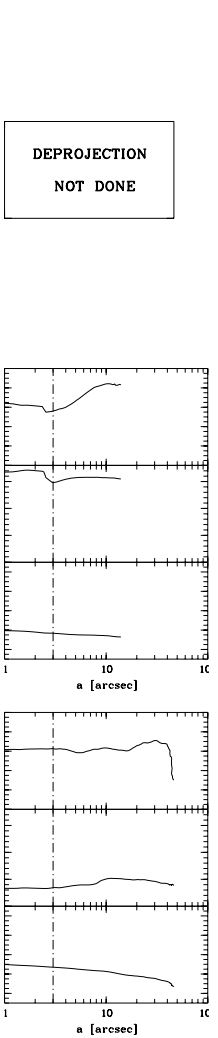

$\mathrm{NGC} 4856(\mathrm{H})$
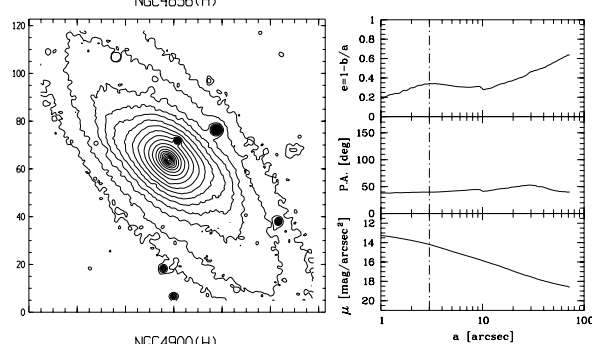

DEPROJECTION

NOT DONE

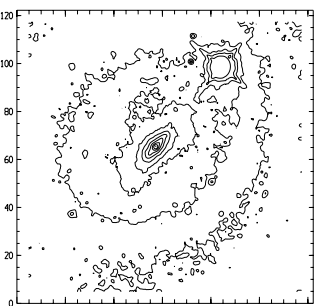

NGC4902(H)

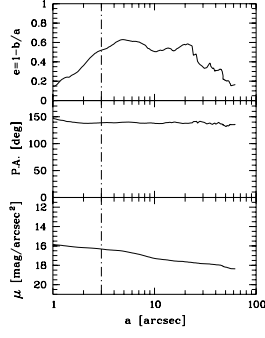

DEPROJECTION NOT DONE

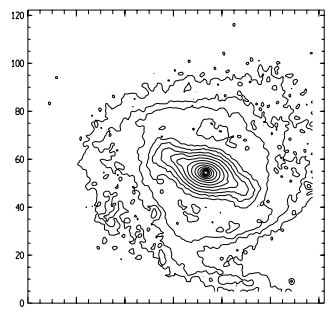

NGC 4984(H)
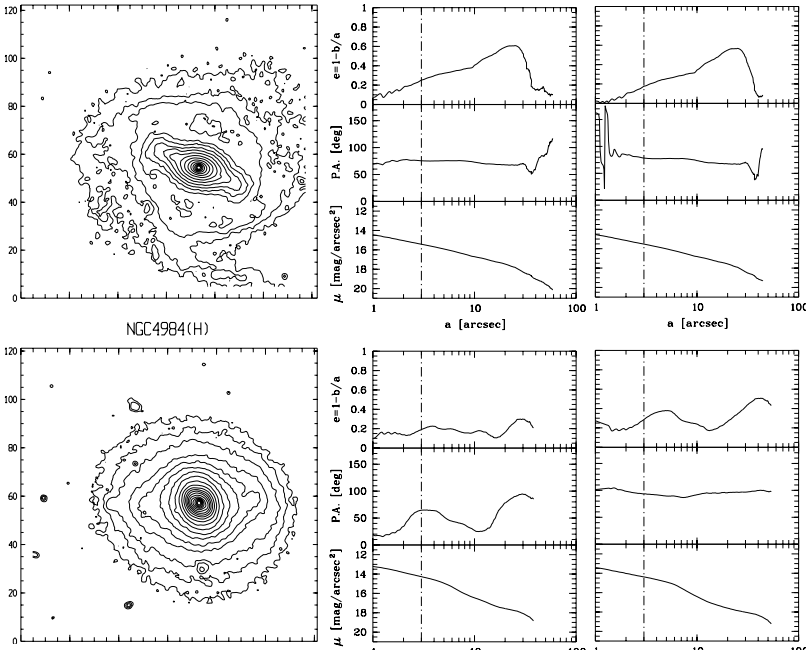

$\underset{\text { a [arcsec] }}{10}$

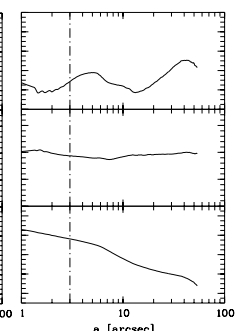

NGC5101(H)
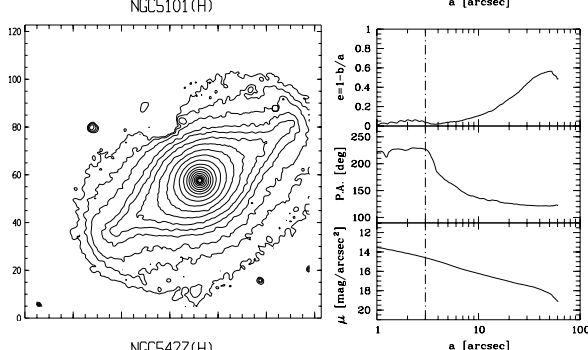

DEPROJECTION

NOT DONE
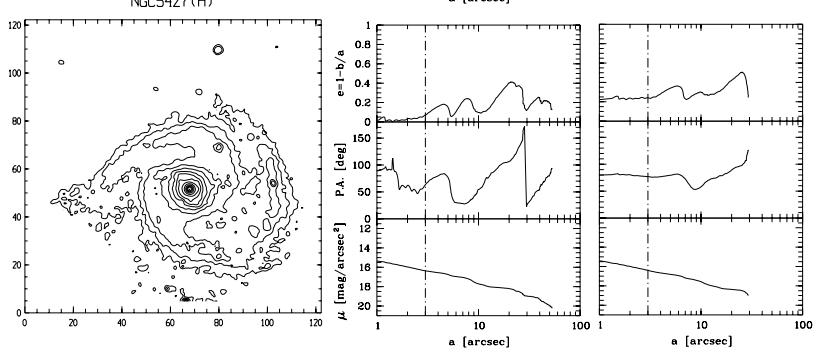

Fig. 4. continued 

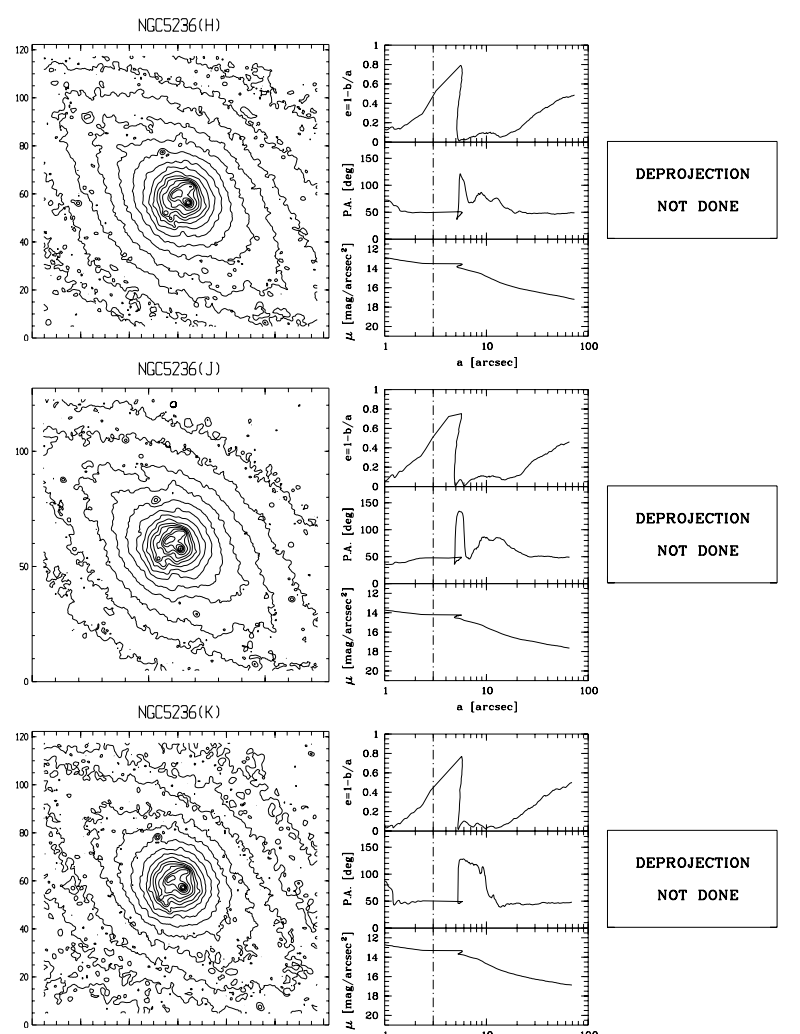

$\mathrm{NCC5566(H)}$
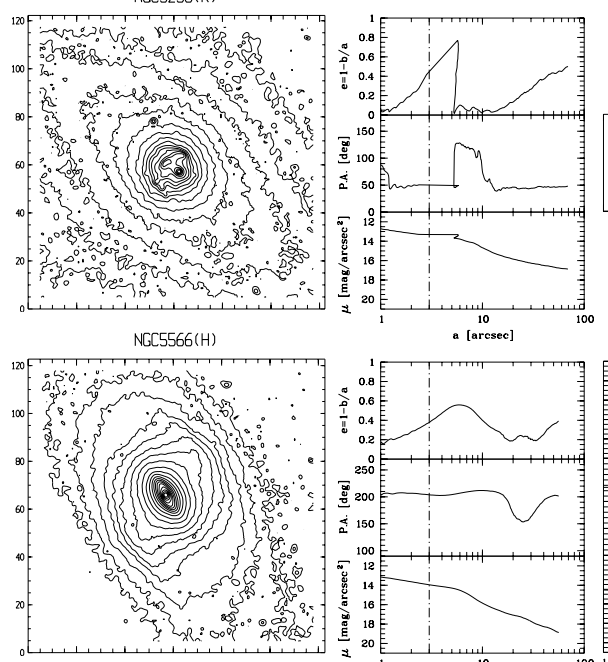

NGC5643(H)

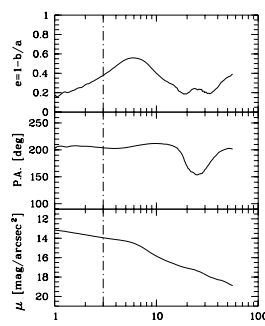

a $\left[\begin{array}{c}10 \\ \text { arcsec }]\end{array}\right.$
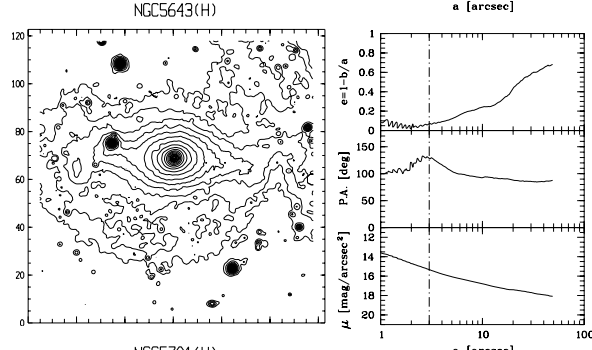

NGC5701(H)
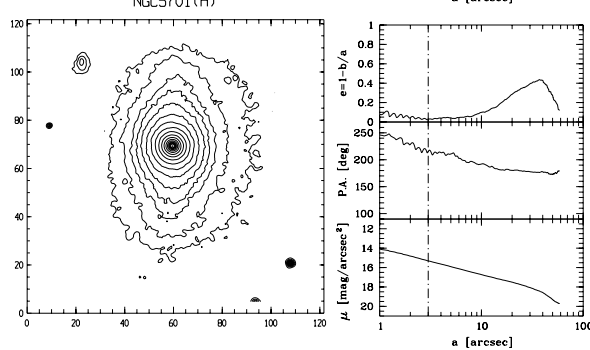

DEPROJECTION NOT DONE

DEPROJECTION

NOT DONE

a [arcsec]
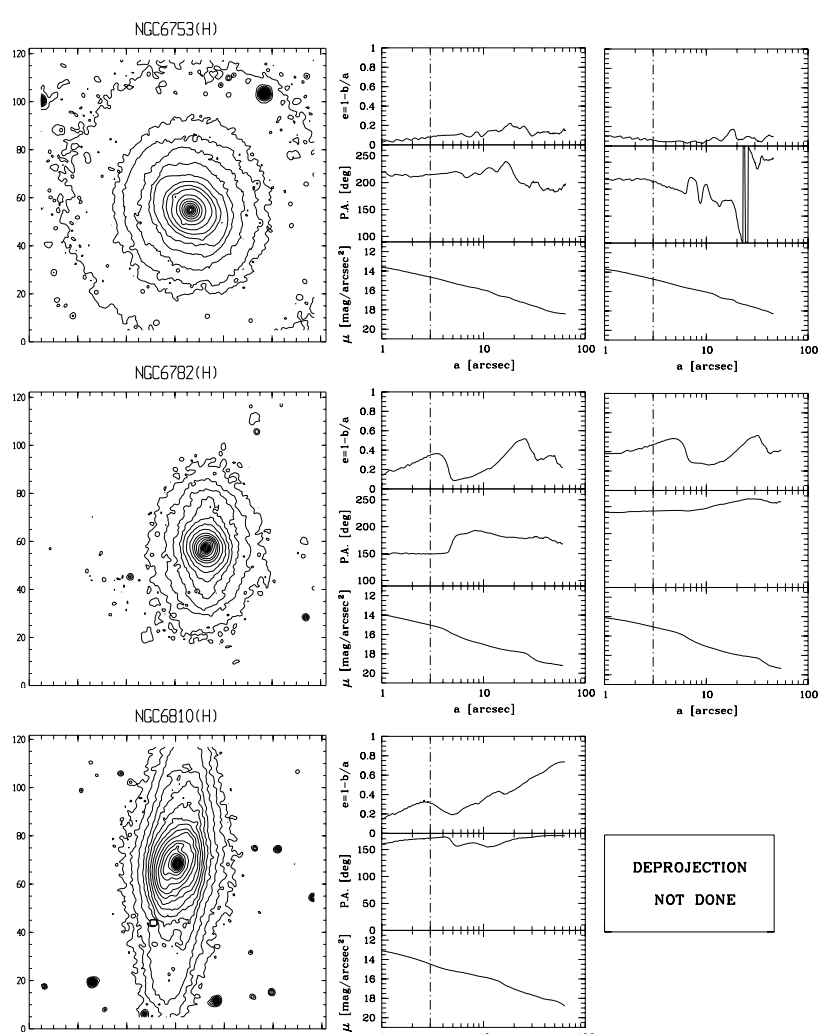

ES0437-67(H)

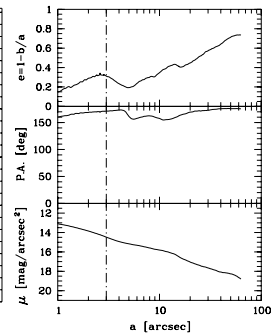

DEPROJECTION NOT DONE
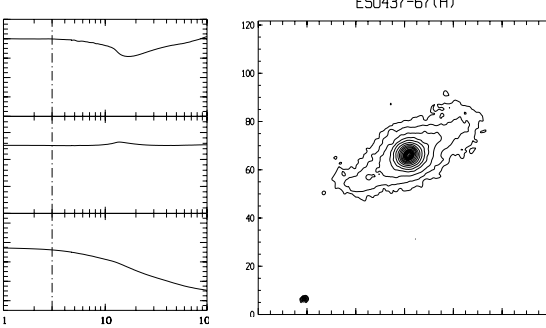

IC1953(H)

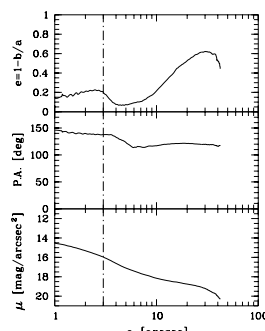

DEPROJECTION NOT DONE
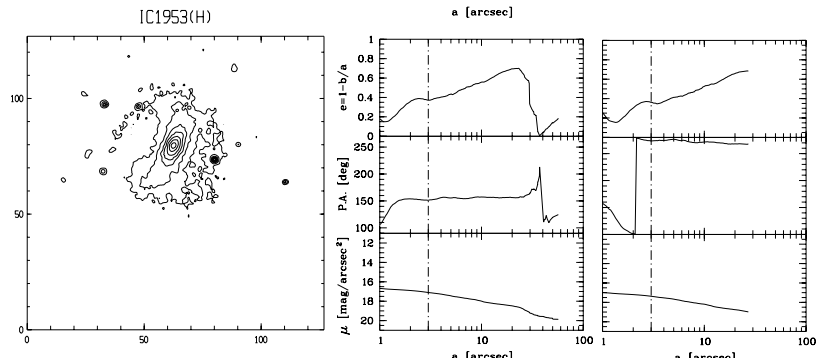

Fig. 4. continued 